\title{
Janus particles: from concepts to environmentally friendly materials and sustainable applications
}

\author{
Claudia Marschelke ${ }^{1,2} \cdot$ Andreas Fery $^{1,2}$ (D) Alla Synytska ${ }^{1,2}($ ) \\ Received: 2 December 2019 / Revised: 9 January 2020 / Accepted: 9 January 2020 / Published online: 4 February 2020 \\ (C) The Author(s) 2020
}

\begin{abstract}
Janus particles represent a unique group of patchy particles combining two or more different physical or chemical functionalities at their opposite sides. Especially, individual Janus particles (JPs) with both chemical and geometrical anisotropy as well as their assembled layers provide considerable advantages over the conventional monofunctional particles or surfactant molecules offering (a) a high surface-to-volume ratio; (b) high interfacial activity; (c) target controlling and manipulation of their interfacial activity by external signals such as temperature, light, $\mathrm{pH}$, or ionic strength and achieving switching between stable emulsions and macro-phase separation; (d) recovery and recycling; (e) controlling the mass transport across the interface between the two phases; and finally (f) tunable several functionalities in one particle allowing their use either as carrier materials for immobilized catalytically active substances or, alternatively, their site-selective attachment to substrates keeping another functionality active for further reactions. All these advantages of JPs make them exclusive materials for application in (bio-)catalysis and (bio-)sensing. Considering "green chemistry" aspects covering biogenic materials based on either natural or fully synthetic biocompatible and biodegradable polymers for the design of JPs may solve the problem of toxicity of some existing materials and open new paths for the development of more environmentally friendly and sustainable materials in the very near future. Considering the number of contributions published each year on the topic of Janus particles in general, the number of contributions regarding their environmentally friendly and sustainable applications is by far smaller. This certainly pinpoints an important challenge and is addressed in this review article. The first part of the review focuses on the synthesis of sustainable biogenic or biocompatible Janus particles, as well as strategies for their recovery, recycling, and reusability. The second part addresses recent advances in applications of biogenic/biocompatible and non-biocompatible JPs in environmental and biotechnological fields such as sensing of hazardous pollutants, water decontamination, and hydrogen production. Finally, we provide implications for the rational design of environmentally friendly and sustainable materials based on Janus particles.
\end{abstract}

Keywords Janus particles $\cdot$ Sustainability $\cdot$ Biotechnology $\cdot$ Environment $\cdot$ Application $\cdot$ Recovery $\cdot$ Green chemistry

\begin{tabular}{llll} 
Abbreviations & & DPV & Differential pulse voltammetry \\
AIE & Aggregation-induced emission & DTT & Dithiothreitol \\
ATRP & Atom transfer radical polymerization & EDX & Energy-dispersive X-ray spectroscopy \\
CAT & Catalase & ELISA & Enzyme-linked immunosorbent assay \\
ConA & Concanavalin A & HDA & 1,6-Hexanediamine \\
DMSA & meso-2,3-di-Mercaptosuccinic acid & JFR & Janus fiber rod \\
DPGG & 1,2-Dipalmitoyl-sn-glycero-3-galloyl & JP & Janus particle \\
& & LSPR & Localized surface plasmon resonance \\
\hline \multirow{2}{*}{$\begin{array}{l}\text { Alla Synytska } \\
\text { synytska@ipfdd.de }\end{array}$} & MBIA & 2-Mercaptobenzoimidazole-5-carboxylic acid \\
1 & MCH & Mercaptohexanol \\
Leibniz-Institut für Polymerforschung Dresden e.V, Hohe Str. 6, & NP & Nanoparticle \\
01069 Dresden, Germany & OPH & Organophosphorus hydrolase \\
2 & OTA & Ochratoxin A \\
Fakultät Mathematik und Naturwissenschaften, Technische & PAA & Poly(acrylic acid) \\
Universität Dresden, 01062 Dresden, Germany & PBDE & Polybrominated diphenyl ether
\end{tabular}




$\begin{array}{ll}\text { PCL } & \text { Polycaprolactone } \\ \text { PCR } & \text { Polymerase chain reaction } \\ \text { PDA } & \text { Polydiacetylene } \\ \text { PDMAEMA } & \text { Poly(2-dimethylaminoethyl methacrylate) } \\ \text { PDEAEMA } & \text { Poly(2-diethylaminoethyl methacrylate) } \\ \text { PDVB } & \text { Polydivinylbenzene } \\ \text { PEG } & \text { Poly(ethylene glycol) } \\ \text { PEO } & \text { Poly(ethylene oxide) } \\ \text { PLA } & \text { Poly(lactic acid) } \\ \text { PLGA } & \text { Poly(lactic-co-glycolic acid) } \\ \text { PLL } & \text { Poly(L-lysine) } \\ \text { PNIPAM } & \text { Poly }(N, N \text {-isopropylacrylamide) } \\ \text { PS } & \text { Polystyrene } \\ \text { PSMA } & \text { Polystyrene-co-maleic anhydride } \\ \text { SDS } & \text { Sodium dodecyl sulfate } \\ \text { SEM } & \text { Scanning electron microscopy } \\ \text { SH-CP } & \text { Thiolated capture probe } \\ \text { TEM } & \text { Transmission electron microscopy } \\ \text { TPE } & \text { Tetraphenylethene } \\ \text { TPEC } & \text { Tetraphenylethene cyanuric chloride }\end{array}$

\section{Introduction}

\section{Janus particles}

Janus particles (JPs), named after the two-faced god Janus from Roman mythology, represent a unique class of patchy particles and synergize two different functionalities at their opposite sides (Fig. 1a). They can be divided into three subgroups of hard (inorganic), soft (organic or polymeric), and hybrid (organic/inorganic) JPs. Apart from conventional spherical shapes, many other geometries of JPs, such as dumbbell-like [2-5], snowman-like [6-13], platelet-like [14-16], or rod-like [17, 18] shapes, have been successfully obtained. Casagrande et al. first applied the term "Janus beads" in 1988 to describe the behavior of amphiphilic beads at water/oil interfaces [19]. Pierre-Gilles de Gennes hypothesized about the potential of Janus grains in his Nobel Prize lecture [20]. During the past 20 years, the research on JPs has prospered (Fig. 1b,c) including manifold synthetic routes for the fabrication of JPs, investigation of their properties, computer simulations as a new perspective to investigate perfect monodisperse Janus particles [21-24], and their potential for various application fields [25].

Individual JPs with both chemical and geometrical anisotropy as well as their assembled layers benefit from their remarkable advantages over the conventional monofunctional particles or surfactant molecules. Due to their anisotropic character, JPs can offer amphiphilic surface properties and a high interfacial activity, so they are also known as solid or colloidal surfactants. The interfacial activity of amphiphilic JPs can be controlled and manipulated by external signals such as temperature, light, $\mathrm{pH}$, or ionic strength by implementation of stimuli-responsive materials to achieve a switching between stable emulsions and macro-phase separation. The stimuli-triggered separation enables the recovery and reusability of JP-based system, besides the use of magnetic components in the design of JPs. Furthermore, the mass transport across the interface between the two phases can be carefully controlled by the size, edge geometry, and porosity of the employed JPs. Finally, the combination of several tunable functionalities in one JP allows their use either as carrier material for the immobilization catalytically active substances or, alternatively, their site-selective attachment to substrates keeping the second hemisphere active for further reactions. All these advantages make JPs a new generation of intelligent building block for the design of advanced materials.

\section{Janus particles as sustainable materials for environmental and biotechnological applications}

Humanity faces severe challenges for the next decades, i.e., climate change, water shortage, loss of biodiversity, soil
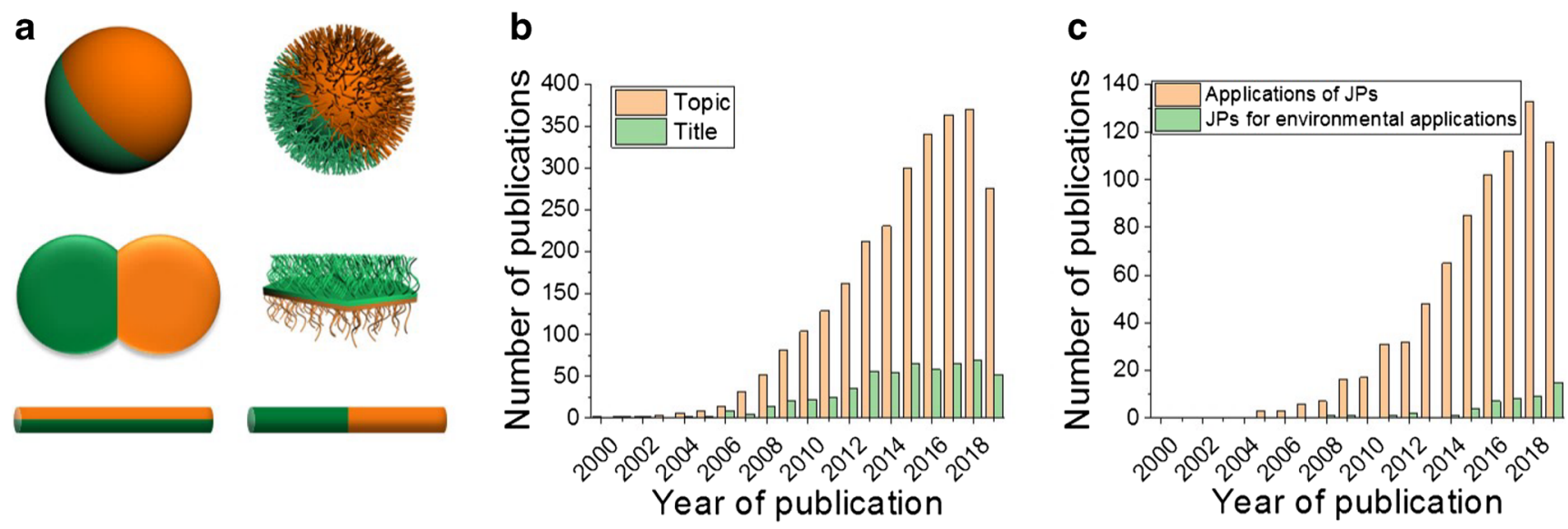

Fig. 1 a Schematics of hairy and not-hairy Janus particles of various geometries synthesized up to date. Number of publications published each year since 2000 on the keywords Janus particles (b), and (environmental) applications of JPs (c). Data taken from ref. [1] 
degradation, and shortage of raw materials. Politics starts to react to these issues, e.g., European development policy comprises the 2030 Agenda for sustainable development with the goal of a sustainable Europe by 2030 . Thus, ideas to realize this aim are highly supported in various research programs. Chemistry can contribute to a more sustainable world by considering aspects of the "Green chemistry." Green chemistry comprises 12 principles that aim at reducing or eliminating the use or generation of hazardous substances in the design, manufacture, and application of chemical products: waste prevention instead of remediation, atom efficiency, less hazardous materials, safer products by design, innocuous solvents and auxiliaries, energy efficient by design, preferably renewable raw materials, shorter synthesis, catalytic reagents, products for degradation, analytical methods for pollution prevention, and inherently safer processes [26].

Although there is a growing awareness of "sustainability" in society, politics, economy, and research, the full application potential of JPs has not been tapped yet in the context of green and sustainable materials. Therefore, in this review, we want to shift the focus from JPs as innovative materials to JPs as an approach for environmental applications and sustainable materials to reinforce the perception of JPs in terms of sustainability. The first part of the review summarizes sustainable biogenic and biocompatible materials used for the synthesis of JPs, as well as recovery, recycling, and reusability strategies for JPs. In the second part of this review, we will discuss the potential of JPs for environmental and biotechnological application fields, which are sensing of hazardous pollutants, water decontamination, and hydrogen production.

\section{Aspects of environmental sustainability}

\section{Bio-based Janus particles: biocompatibility and biodegradability of Janus particles}

To avoid any negative impact of the JPs on the environment, non-hazardous or even biogenic materials should be preferred for the JP synthesis especially in environmental and biological contexts. The utilization of bio-based materials for the synthesis of JPs meets some requirements of the green chemistry concept, such as the use of less hazardous materials, generation of safer products by design, and employment of preferably renewable raw materials [26]. Therefore, several groups aim for the design of JPs based on different bio-based materials (Table 1). Polysaccharides, such as alginates [27-34, 38], chitosan [32, 35, 36, 40, 54-57], pectin [38], cellulose [39], and heparin [40], are a very often used group of biogenic materials. Further substance classes such as polypeptides [41] or inorganic compounds [32, 42] have not been extensively used yet.
Apart from biogenic materials that provide a certain biocompatibility and degradability by nature, there are also few synthetic biocompatible and biodegradable materials used for JP synthesis and application present in literature. The most commonly used materials are poly(lactic acid) (PLA), poly(lactic-co-glycolic acid) (PLGA), and polycaprolactone (PCL). These synthetic polyesters are FDA-approved, biodegradable polymers that are frequently used in drug delivery systems (Table 1).

Soft, organic JPs are the largest group among biogenic and biocompatible JPs comprising microgels and microcapsules. One of the most common techniques used for the fabrication of these soft biogenic or biocompatible JPs is the microfluidic approach that provides monodisperse JPs with diameters ranging from tens to hundreds of micrometers. Droplet-based microfluidic processes for the fabrication of anisotropic microgels have been accomplished using w/o emulsion droplets combined with UV-induced polymerization or ionic crosslinking to solidify the Janus droplets (on-chip or offchip) [28]. The design of the fabrication process broadly varies from (double) T-junction microfluidic devices [27, 36, 58], stop-flow lithography microfluidic techniques [59, 60], flow-focusing microfluidic devices [30, 33, 38, 44, 61], double emulsion droplet templates [62], and single emulsion/offchip cross-linking [28]. Using this technique, magnetic and/or fluorescent components can be easily embedded in one compartment of the JPs $[27,30,34,36]$.

Further techniques for the fabrication of biogenic JPs follow very diverse approaches, such as electrohydrodynamic co-jetting [45], double emulsion solvent evaporation [46], single-step solvent emulsion [43], and polymerization [52] and template-based methods [32], and are rather hardly represented in the literature.

\section{Recovery and recycling of Janus particles}

Recovery and recycling of materials are further key aspects for environmental sustainability, therefore also for JPs. "Recovery" describes the process of removing a material for (re-)use; however, "recycling" refers to the process of converting a waste material into a new one. Nonetheless, both aspects are key issues of modern waste reduction and aim at the environmental sustainability and the concept of green chemistry [26]. JPs offer problematic concerns, but also large potential for their recovery and recycling. On the one hand, nano- and micro-sized (Janus) particles can be hardly retained by conventional technologically used filter systems and would be released to the environment and ground water with so far unknown consequences. On the other hand, JPs feature great potential, i.e., in terms of their multifunctionality, to get separated after their successful use.

One of the easiest methods to recover (Janus) particles from a liquid medium is the centrifugation of these particles, as 
Table 1 Materials and applications of biogenic and biocompatible JPs

\begin{tabular}{|c|c|c|}
\hline Material & Description and application & Ref \\
\hline \multirow[t]{9}{*}{ Alginate } & $\begin{array}{l}\text { Janus calcium alginate hydrogel particles with one magnetic hemisphere for } \\
\text { cell encapsulation via microfluidics }\end{array}$ & $\left.\begin{array}{l}{[27]} \\
{[28}\end{array}\right]$ \\
\hline & $\begin{array}{l}\text { Janus sodium alginate/PNIPAM microgels via microfluidics with external } \\
\text { ionic crosslinking }\end{array}$ & {$[29]$} \\
\hline & $\begin{array}{l}\text { Bi- and triphasic alginate particles with embedded polydiacetylene } \\
\text { liposomes for multitargeting detection }\end{array}$ & {$[31]$} \\
\hline & Magnetic-fluorescent calcium alginate JPs doped with $\mathrm{Fe}_{3} \mathrm{O}_{4} \mathrm{NPs}$ and & {$[32]$} \\
\hline & $\mathrm{CdSe} / \mathrm{ZnS}$ quantum dots via microfluidics for biomolecular detection & {$[33]$} \\
\hline & $\begin{array}{l}\text { Sodium alginate multicompartmental particles via centrifuge-based droplet } \\
\text { shooting device }\end{array}$ & [34] \\
\hline & Alginate-chitosan Janus hydrogel particles & \\
\hline & $\begin{array}{l}\text { Sodium alginate multicompartmental JPs via multiplex coaxial } \\
\text { flow-focusing process }\end{array}$ & \\
\hline & $\begin{array}{l}\text { Alginate Janus hydrogel microparticles via microfluidics for sensing of } \\
\text { glucose and cholesterol }\end{array}$ & \\
\hline \multirow[t]{3}{*}{ Chitosan } & Chitosan-capped silver JPs with antimicrobial activity & {$[35]$} \\
\hline & Magnetic chitosan- $\mathrm{Fe}_{3} \mathrm{O}_{4}$ Janus dimer capsules via microfluidics & {$[36]$} \\
\hline & $\begin{array}{l}\text { Chitosan microcapsules containing FePt NPs as microcarriers prepared via } \\
\text { microfluidics }\end{array}$ & {$[37]$} \\
\hline Pectin & Pectin-pectin and pectin-alginate Janus microbeads via microfluidics & {$[38]$} \\
\hline Cellulose & $\begin{array}{l}\text { Hairy Janus cellulose nanocrystalloids by hydrolysis of disordered cellulose } \\
\text { chains of hierarchical fiber structures }\end{array}$ & {$[39]$} \\
\hline Heparin & $\begin{array}{l}\text { Chitosan-heparin capsules via layer-by-layer technique partially coated with } \\
\text { a gold layer for photothermal thrombus therapy }\end{array}$ & {$[40]$} \\
\hline Poly(lysine) & $\begin{array}{l}\text { Self-propelled Janus swimmers modified with PLL, PEGylated PLL, Pt } \\
\text { NPs, and glucose oxidase }\end{array}$ & {$[41]$} \\
\hline Inorganic components & $\begin{array}{l}\text { Seawater-driven magnesium Janus micromotors with a nickel-gold bilayer } \\
\text { patch for environmental applications }\end{array}$ & {$[42]$} \\
\hline \multirow[t]{3}{*}{ Poly(lactic acid) (PLA) } & PLA- and PLGA-containing JPs via single-step solvent emulsion technique & {$[43]$} \\
\hline & for encapsulation of therapeutic and diagnostic agents & {$[44]$} \\
\hline & $\begin{array}{l}\text { PLA-PLGA JPs via flow-focusing glass microfluidic devices for drug de- } \\
\text { livery }\end{array}$ & \\
\hline \multirow{7}{*}{$\begin{array}{l}\text { Poly(lactic-co-glycolic } \\
\text { acid) (PLGA) }\end{array}$} & Multicompartmental colloids made from PLGA via electrohydrodynamic & {$[45]$} \\
\hline & co-jetting & [46] \\
\hline & PLGA- and precirol-based polymer-lipid JPs via water-in-oil-in-water & {$[47]$} \\
\hline & $\begin{array}{l}\text { double emulsion solvent evaporation technique as carriers for anticancer } \\
\text { drugs }\end{array}$ & {$[48]$} \\
\hline & PLGA-based JPs via solvent evaporation method & [49] \\
\hline & $\begin{array}{l}\text { PLGA-based Janus NPs prepared via fluidic nanoprecipitation system for } \\
\text { encapsulation of anticancer drugs }\end{array}$ & \\
\hline & $\begin{array}{l}\text { Magnetic-fluorescent Janus PLGA microspheres with rare earth ions via } \\
\text { electrospraying }\end{array}$ & \\
\hline \multirow[t]{5}{*}{ Polycaprolactone (PCL) } & Patchy JPs composed of polycaprolactone and PLA via microfluidics & {$[50]$} \\
\hline & Complex-shaped PCL/PLGA microparticles via microfluidics & {$[51]$} \\
\hline & pH-responsive hollow PCL-PDEAEMA Janus containers by ring-opening & {$[52]$} \\
\hline & and atom transier radical polymerization ror arug denvery & {$[53]$} \\
\hline & PCL/PLGA Janus microcapsules via microfluidics for drug delivery & \\
\hline
\end{tabular}

shown by many groups [8, 63-72]. However, this approach lacks in its applicability, since only very limited volumes can be treated. Nevertheless, it could be proved that JPs could be used multiple times as for example by Huang, Qi et al. for enzymatic catalysis (Fig. 2) [65] or by Prasad et al. for dye separation [67]. A further approach is the filtration of (Janus) particles as applied by Chen et al. [73]. This technique benefits from its simple applicability on many size scales, but is limited by the conflict between technologically realizable pore sizes (and related flow rates) and the small dimensions of micro-, submicro-, and nanometer-sized JPs.

Another more advanced possibility is the separation of JPs by magnetic fields, which can be enabled by the use of ferromagnetic materials such as $\mathrm{Fe}_{3} \mathrm{O}_{4}$ or nickel in the 
a

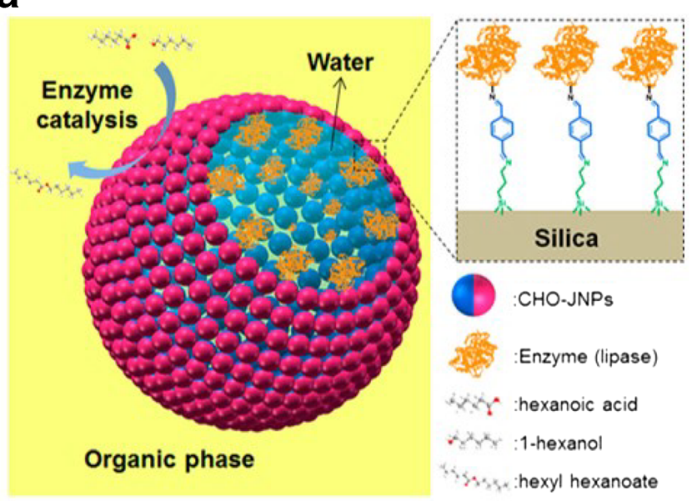

b

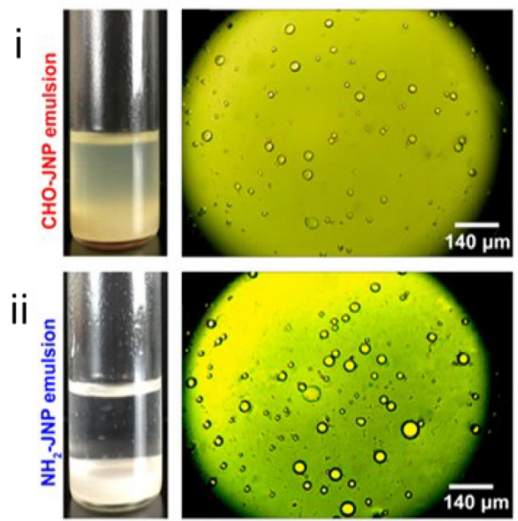

C

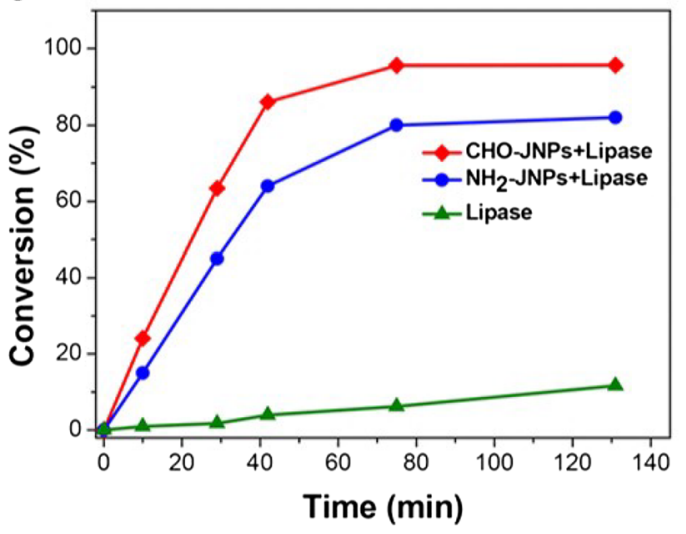

Fig. 2 a Schematic of the esterification reaction of hexanoic acid with 1hexanol in lipase-immobilized CHO-Janus NP Pickering emulsion. b Photographs and optical micrographs of Pickering water-in-heptane emulsions with lipase stabilized by CHO-Janus NPs (i) and $\mathrm{NH}_{2}$-Janus NPs (ii). c Time courses of free lipase, lipase-immobilized CHO- and

design concept of the JPs. As an example, Zhang et al. demonstrated the fast and easy recovery of poly(methylmethacrylate-acrylic acid-divinylbenzene)/ iron oxide JPs, used as demulsifier for heavy crude oilwater emulsions, by employing an external hand magnet without losing its interfacial activity [74]. Rhee, Kim et al. introduced Janus colloid surfactant catalysts with magnetic responsiveness in Pickering emulsion microreactors [75]. The amphiphilic JPs patched with Pd or Ag NPs assembled at the reactant-water interface and enhanced the reaction kinetics and product yields [75]. By co-patching of the JPs with $\mathrm{Fe}_{3} \mathrm{O}_{4} \mathrm{NP}$, the products could be easily separated and the JP catalysts were recovered and reused in up to five cycles without significant loss in reaction conversion [75]. Further examples were published by Xu et al. for catalytically active Janus NPs [76]; Klinger, Hawker, and Kim et al. for $\mathrm{pH}$-switchable JP-based surfactants [77]; Zhang et al. for Janus phase transfer catalysts [78]; and many others [79-84]. Although magnetic separation is an easy and fast technique widely used in research systems, its industrial implementation has not been extensively realized yet.
One example combining reusability aspects with catalytic applications was developed by Synytska et al., who synthesized hybrid hairy JPs with an inorganic silica core (diameter: $200 \mathrm{~nm}$ ) and poly(acrylic acid) (PAA) and polystyrene brush shells at the opposite sides of the core (Fig. 3a) [64]. Catalytic $\mathrm{Ag}$ or Au NPs could be selectively immobilized into the PAA brushes (Fig. 3b) [64]. The hairy polymer shells provided a better distribution of the noble NPs in the polymer, high wettability contrast between the polymers, and stimuliresponsiveness of the system on demand [64]. Thus, the JP catalysts combined efficient pH-responsive (re-)stabilization of o/w emulsions (Fig. 3c) and successful catalytic reduction of eosin $\mathrm{Y}$ at the water-oil interface (Fig. 3d,e) [64].

Recently, a different approach for the separation of JPs was developed by the same group [85]. Bifunctional hybrid hairy PDMAEMA/PNIPAM JPs utilized for enzyme immobilization and dye-contaminated waste water treatment were separated by temperature-induced flocculation and sedimentation within $15 \mathrm{~min}$ [85]. At elevated temperatures above $40^{\circ} \mathrm{C}$, the thermoresponsive PNIPAM brushes collapsed leading to hydrophobization of the JPs and, thus, to their aggregation. 
a

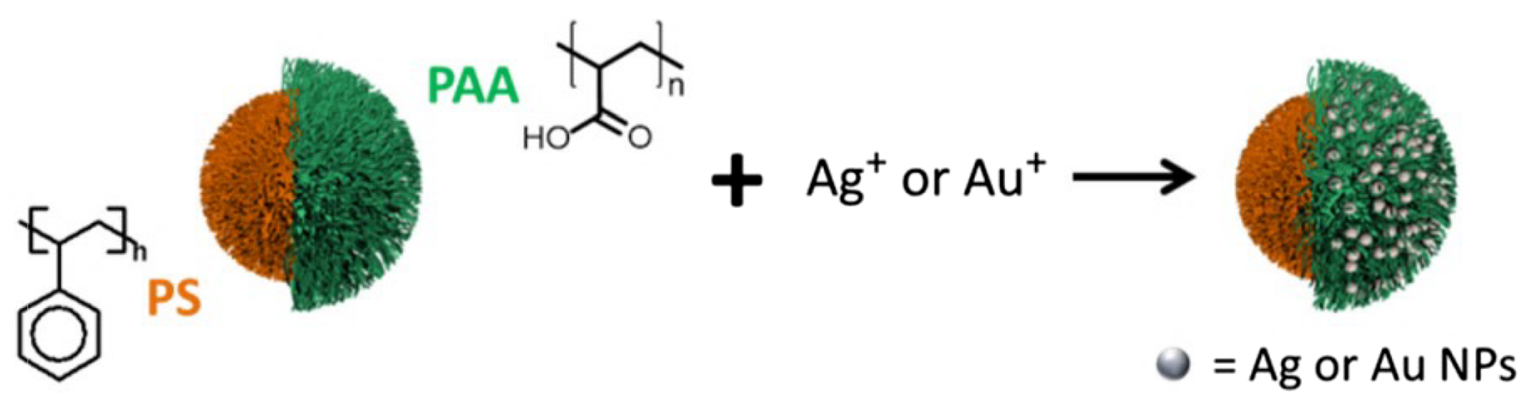

b

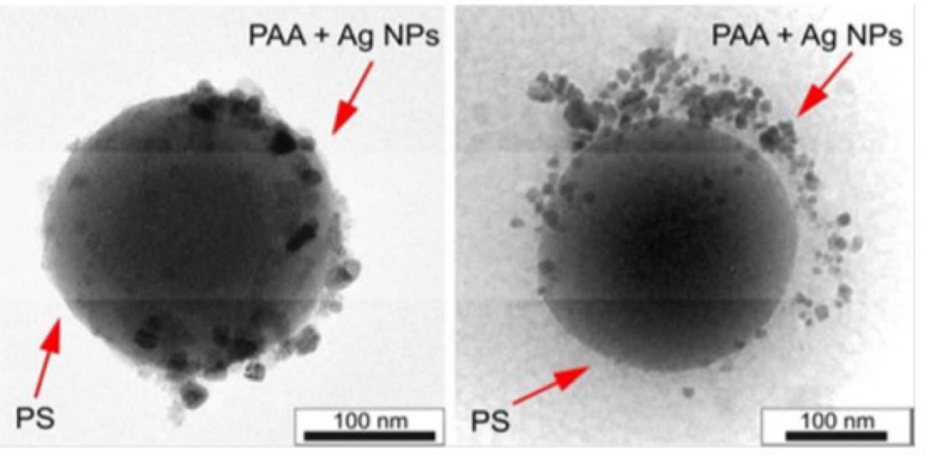

C

PAA/PS-Ag-JP, $\mathrm{pH}=7$

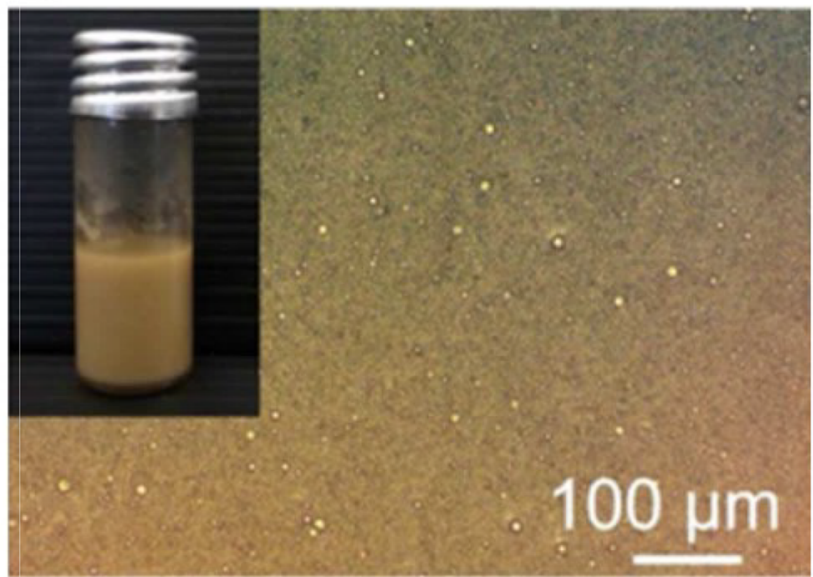

d
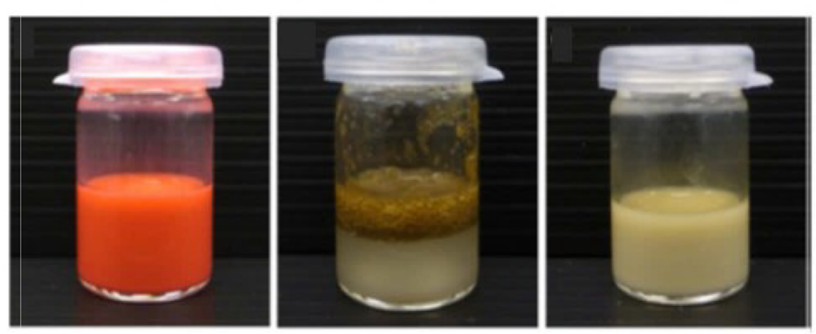

Fig. 3 a Scheme of the selective NP immobilization onto PAA/PSmodified hybrid hairy JPs. b Representative TEM and cryo-TEM images of the JPs with selectively immobilized Ag NPs. c Photographs (insets) of the JP-stabilized emulsions at $\mathrm{pH} 7$ (left, stabilized) and $\mathrm{pH} 2$ (right, destabilized). $\mathbf{d}$ Photographs taken before and after the reduction of eosin
PAA/PS-Ag-JP, $\mathrm{pH}=2$

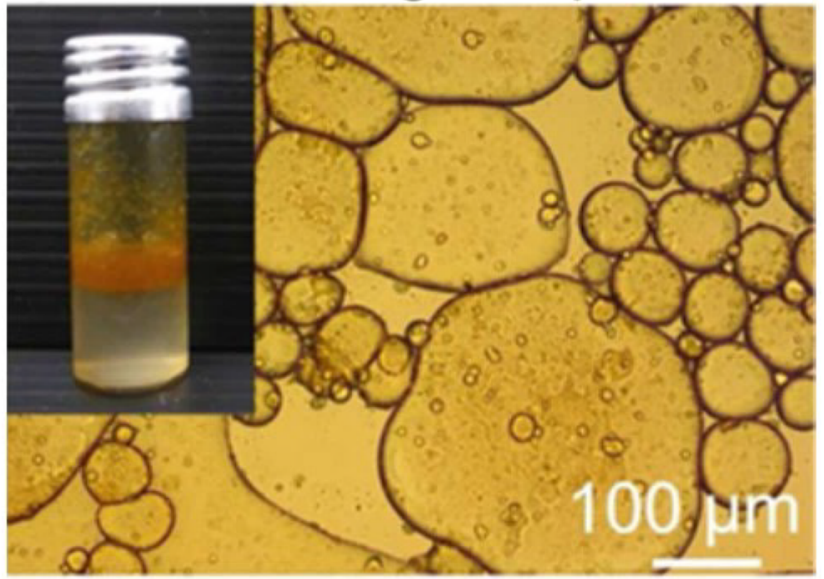

$\mathbf{e}$

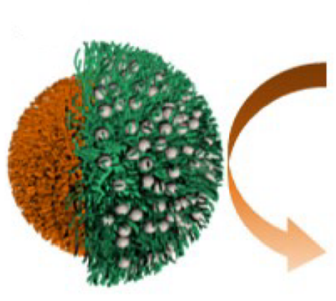

$\mathrm{COO}_{\mathrm{Br}}$ Br orangered

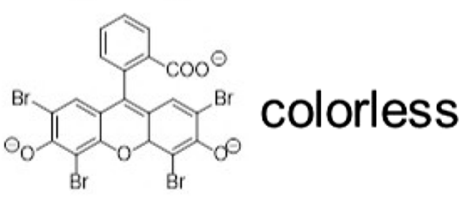

$\mathrm{Y}$ in an $\mathrm{o} / \mathrm{w}$ emulsion stabilized by the JP catalysts. After the catalytic reaction, the emulsion can be restabilized. e Scheme of the Ag-decorated JP-catalyzed eosin Y reduction. Reproduced with permission from ref. [64]. Copyright 2015 American Chemical Society 
Subsequently, the supernatant could be removed and the JPs could be reused for enzymatic catalysis with only minor losses in enzymatic activity (2-5\%) [85].

\section{Aspects of environmental application}

In this chapter, we will address the tenth and the twelfth principle of green chemistry, based either on bio-based natural polymers or on fully synthetic biocompatible and biodegradable ones, which aim at the design of products for degradation and methodologies for pollution prevention, respectively [26]. Therefore, we introduce JP-based systems for water decontamination, environmental sensing, and production of hydrogen as alternative energy source. The focus of this chapter is rather placed on environmental and biotechnological applications than on the biocompatibility of the materials used for the JPs.

\section{Sensing and monitoring applications}

Sensing and monitoring in environmental contexts denotes the evaluation of water or food quality by measuring chemical, physical, or biological parameters [86]. The main objectives of environmental sensing are to monitor the current status of an environment, to warn about incoming hazardous changes, and to control remediation processes [86]. Sensing and monitoring approaches face several challenges, namely high sensitivity and specificity towards very low concentrations of contaminants, stability in complex natural media, and fast response times [87]. JPs may contribute to novel concepts of effective sensing and monitoring of various pollutants, which will be reviewed in the following subchapter.

\section{Metal detection}

In 2014, Kim and coworkers demonstrated 500- $\mu$ m Janus-compartmental alginate microbeads for selective and facile colorimetric detection of lead(II) [88]. These JPs are composed of two divided phases of label-free sensory polydiacetylene (PDA) liposomes and magnetic NPs in an alginate hydrogel matrix [88]. The liposomes contain 1,2-dipalmitoyl-snglycero-3-galloyl lipids (DPGG) whose galloyl headgroups can form phenolic metal complexes with lead(II) [88]. The formed inter- and intraliposomal interactions induced distortion of the conjugated "yne-ene" main chain of PDA and produced a strong colorimetric signal with a color change from blue to red and a red fluorescence [88]. The alginate matrix also allows simultaneous removal of lead(II) ions via adsorption [88]. For easy handling during washing and detection process, the JPs can be magnetically manipulated [88].

A further interesting example of JP-based metal detection was shown by Yoon et al. in 2018 [89]. They reported on streptavidin-modified retroreflective JPs employed for a highly selective and sensitive optical sensing platform for mercury ions with a detection limit of $0.027 \mathrm{nM}$ [89]. In the presence of mercury ions, a biotinylated stem-loop DNA probe formed a double-stranded structure with the loop region via mercury-mediated thymine-thymine stabilization, which led to stretching of the DNA probe and exposition of the biotin moiety [89]. The biotin interacts with the streptavidin-modified JPs, which translate the $\mathrm{Hg}^{2+}$-structural changes in the DNA probe into a measurable optical signal [89]. Since the number of biospecifically bound JPs is proportional to the mercury concentration, the concentration of $\mathrm{Hg}^{2+}$ could be also quantified between 0 and $100 \mathrm{nM}$ of $\mathrm{Hg}^{2+}$ [89].

Recently, $\mathrm{Xu}$ et al. developed a facile microfluidic fabrication route for the synthesis of hybrid Au nanorod@Ag polyaniline Janus nanoparticles, which can be employed as a surface-enhanced Raman scattering sensor for the detection of mercury ions with good selectivity and sensitivity due to strong interactions between polyaniline and $\mathrm{Hg}^{2+}$ ions [90]. The coordination of the nitrogen atoms of polyaniline and $\mathrm{Hg}^{2+}$ ions reduced the stability of the JPs and caused aggregation between the JPs [90]. Thus, the resulting SERS enhancement is linearly dependent on the $\mathrm{Hg}^{2+}$ concentration within a range of 1-150 $\mathrm{nM}$ [90].

\section{DNA detection}

In 2010, Wang et al. proposed synthetic Au-Pt nanomotors, which were able to detect DNA and bacterial ribosomal RNA (Fig. 4) [91]. This fast, simple, and sensitive DNA-sensing concept is based on measuring changes in the speed of catalytic nanomotors caused by the dissolution of Ag NP captured in a sandwich DNA hybridization assay [91]. In presence of target DNA, the nucleic acid target formed a duplex with a thiolated DNA capture probe and a Ag NP detector probe [91]. The Ag NP tags dissolve in the hydrogen peroxide fuel solution [91]. Subsequently, the released silver ions caused a significant increase in the speed of the added nanomotors (Fig. 4a) [91]. The sensing approach is also a quantitative concept: the higher the concentration of the nucleic acid target, the more Ag NPs are captured and releasing $\mathrm{Ag}^{+}$ions, and the faster the movement of the nanomotors (Fig. 4b) [91].

Baeza, Vallet-Regí et al. demonstrated a different, biocatalyzed approach of DNA sensing using self-propelled micro/nanomotors [92]. The synthesized mesoporous silica particles were asymmetrically modified with catalase and single-stranded DNA [92]. Catalase enabled the swimming performance by generating oxygen bubbles in a diluted hydrogen peroxide solution [92]. The single-stranded DNA selectively captured a DNA-functionalized micrometric cargo particle [92]. The DNA strands are not complementary to each other, but they are complementary to one of the terminal 


\section{a}

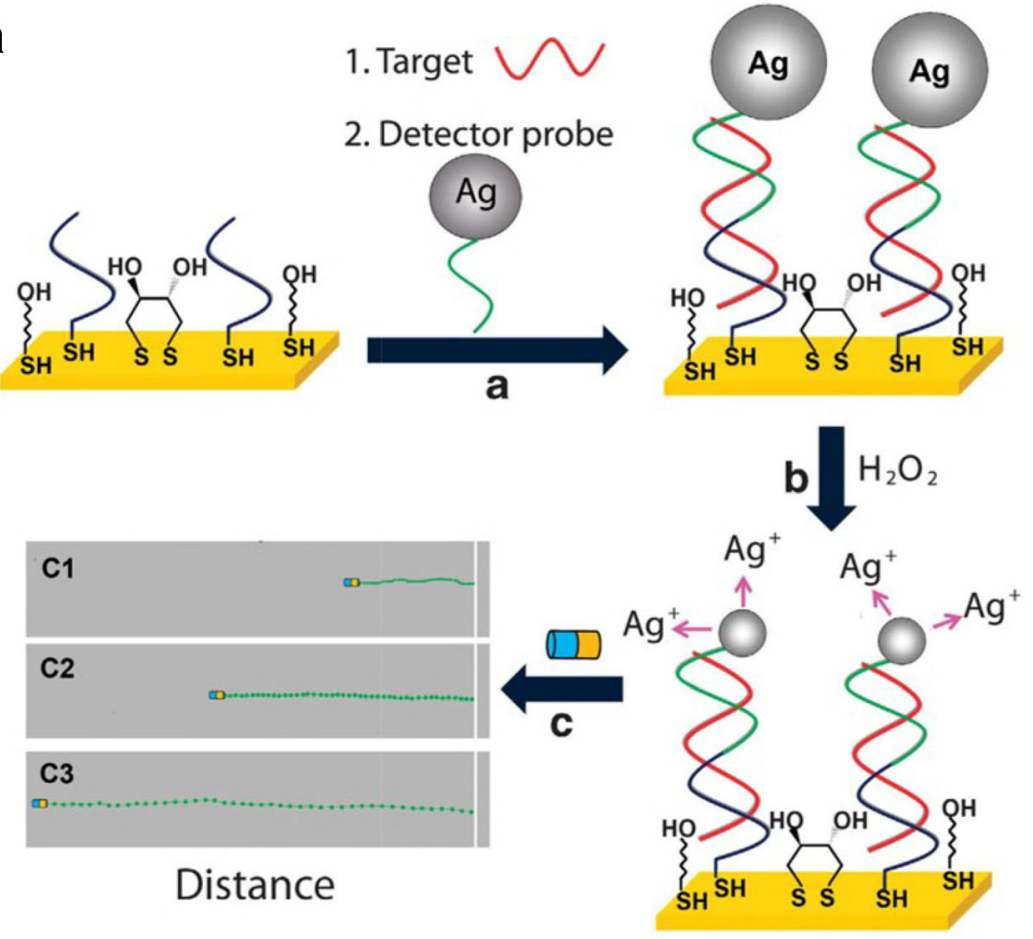

b

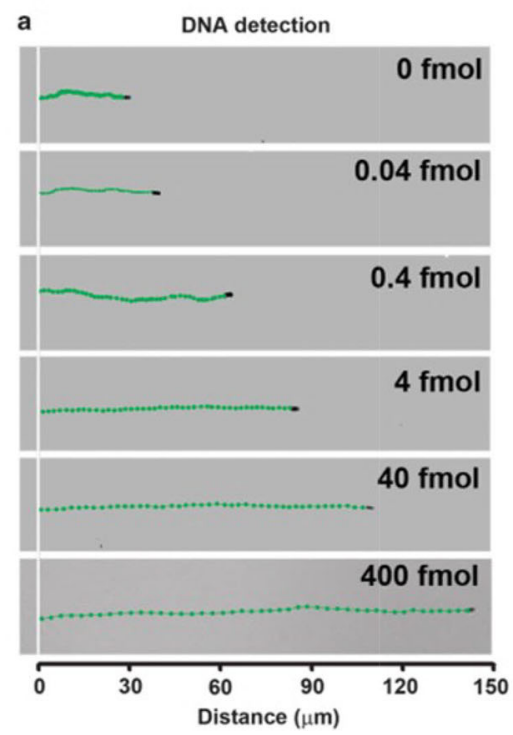

Fig. 4 a Motion-based nucleic acid detection: hybridization of the target and capture of the Ag NP-tagged detector probe in a typical sandwich assay on the ternary SH-CP/DTT+MCH surface; dissolution of Ag NP tags in the peroxide fuel leads to $\mathrm{Ag}^{+}$-enriched fuel; visual detection of the motion of the catalytic nanowire motors in the $\mathrm{Ag}^{+}$-enriched fuel with increasing target nucleic acid concentrations from $\mathrm{C} 1$ to $\mathrm{C} 3$. b

fragments of a larger analyte strand that serves as a highly selective bridge between the particles [92]. Only in case of hybridization of both sides of the analyte DNA the motor was connected to the cargo system and movement could be detected [92]. Thus, the presented hybrid nanomotors are able to detect, capture, and isolate DNA in biological samples [92].

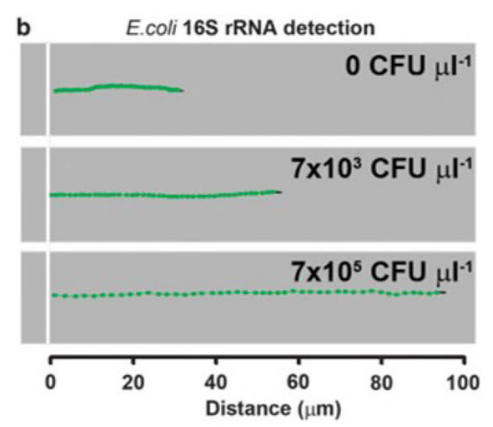

Quantitative nucleic acid detection: motion-based DNA detection of different levels of synthetic target and E. coli $16 \mathrm{~S}$ rRNA corresponding to different cell concentrations. Optical images superimposed with straightline track lines showing the distance covered by the corresponding Au$\mathrm{Ni}$-Au-Pt nanomotors over a period of $4 \mathrm{~s}$. Reproduced with permission from ref. [91]. Copyright 2010 Nature Publishing Group

\section{Bacteria detection}

The fast and sensitive detection of bacteria is indispensable in many fields of application such as food safety, medical diagnostics, water quality, and counter terrorism [93-95]. Foodborne pathogens like Escherichia coli, Salmonella, 
Listeria monocytogenes, and Campylobacter infect millions of people worldwide every year. Commonly, these bacteria are detected using culture techniques, ELISA (enzyme-linked immunosorbent assay), microscopy, or PCR (polymerase chain reaction), which are time-consuming, labor-intensive, and not real-time. Therefore, novel strategies for the rapid, sensitive, reliable, and simple detection of these pathogens is urgently needed.

Li et al. proposed a "motion-capture-lighting" strategy by motion-enhanced capture of bacteria and captured-induced fluorescence of micromotors [95]. They used Janus fiber rods (JFRs) prepared by cryocutting of aligned fibers made by sideby-side electrospinning [95]. To enable bubble propulsion of the Janus fiber rods, catalase was grafted on one side of the fibers to generate oxygen bubbles (Fig. 5a) [95]. The other side was modified with mannose for specific recognition of FimH proteins of fimbriae on bacterial surfaces (Fig. 5a) [95]. Capture of $E$. coli on the mannose-functionalized fiber side led to lighting up of the Janus micromotors due to aggregationinduced emission effect of the incorporated tetraphenylethene derivative (Fig. 5a) [95]. The fluorescence color of the E. colicontaminated Janus micromotor suspensions turned from to blue to green (Fig. 5b) [95]. The bacterial concentrations could be also quantified from the fluorescence intensities (Fig. 5b) [95].

\section{Toxin detection}

Besides the contamination of food and water with bacteria, mycotoxins produced by around 200 different organisms of the fungus kingdom are capable of causing severe diseases to humans and animals. Due to their negative effects on public health, levels of these mycotoxins are strictly regulated in legal requirements of the European Union. Current approaches for mycotoxin detection and determination employing immunoassays and liquid chromatography coupled to tandem mass spectrometry lack in their practical applicability especially in terms of real-time monitoring, complexity, and nonportability [96]. In 2019, two different Janus particle-based approaches were developed for the detection of ochratoxin A (OTA), a toxic mycotoxin produced by several Aspergillus and Penicillium species. Zhao et al. reported on a ratiometric SERS (surface-enhanced Raman scattering) aptasensor for the quantitative detection of ochratoxin A based on plasmonic Au-Ag Janus nanoparticles [97]. The Au-Ag Janus NPs were fabricated via sequential growth of silver islands on gold cores. The ligand 2-mercaptobenzoimidazole-5-carboxylic acid (MBIA) operates as a Raman reporter, while the $\mathrm{Au}-\mathrm{Ag}$ Janus NPs enhance the SERS signal. The combination of these JPs with highly stable MXenes nanosheets allowed the introduction of an internal standard for quantitative analysis of ochratoxin A [97]. The Janus NPs were connected to the MXenes nanosheets via hydrogen bonds and chelation interaction between phosphate groups and titanium ions. In absence of OTA, Raman signal from both MXenes nanosheets and Janus NPs could be detected. In the presence of OTA, the OTA aptamer preferably interacts with the OTA target, leading to the dissociation of the Janus NPs from the MXenes nanosheets and to a decrease of the SERS signal. The amount of OTA could be then quantified based on the linear correlation between the ratiometric peak intensity of $\mathrm{I}_{\text {Janus NP }} / \mathrm{I}_{\text {MXenes }}$ and OTA concentration [97]. This sensitive and reliable approach can be also applied for the quantitative determination of analytes in real systems such as red wine [97].

In another contribution, He, Shuang et al. presented an organic JP-based aptasensor ochratoxin A detection [98]. In this approach, aminated polystyrene particles were coated on one hemisphere with gold by vacuum evaporation. Then, a thiolated ochratoxin aptamer was immobilized onto the gold layer for selective OTA identification. The second hemisphere of the polystyrene particles carrying amino groups on the surface was used to bind the JPs to the surface of a glassy carbon electrode modified with carboxylated graphene via peptide bonds. The sensor was able to detect interactions between aptamer and OTA by differential pulse voltammetry (DPV) with OTA concentrations in the range from $10^{-5}$ to $10 \mathrm{nM}$. The system could be also successfully applied for the determination of OTA contents in red wine samples with high stability and reproducibility [98].

Jurado-Sánchez, Escarpa et al. proposed the use of dynamic Janus micromotors as mobile sensors for the detection of toxins released by enterobacteria as indicators of food contamination [94]. The Janus micromotors were synthesized in a Pickering emulsion process and contain Platinum NP for bubble-propulsion and receptor-functionalized quantum dots for selective binding of 3-doxy-D-manno-oct-2-ulosonic acid target in endotoxin molecules, such as lipopolysaccharides from Salmonella enterica [94]. The fluorescence of the Janus micromotors was rapidly and concentrationdependently quenched when the quantum dots interacted with the target endotoxins [94]. Utilizing this micromotor assay, low endotoxin concentrations of $0.07 \mathrm{ng} \mathrm{mL}^{-1}$ could be detected within $15 \mathrm{~min}$, which is far below the level toxic to humans $\left(275 \mu \mathrm{m} \mathrm{mL}^{-1}\right)$ [94].

\section{Water decontamination}

Water contamination has become a serious global concern since it has harmful effects on the human health and the living environment. Therefore, rapid and efficient solution approaches are urgently required. Toxic contaminants comprise dispersed and dissolved organic and inorganic compounds, e.g., solvents, organic dyes, oil, personal care or consumer products, pharmaceuticals, pesticides, heavy metal ions, bacteria, and microplastics. 
Fig. 5 a Fluorescence response of Janus micromotors after capture of E. coli. Self-propelled Janus micromotors were prepared by modification with TPEC-Man on one side and CAT on the other side of JFRs. The decomposition of $\mathrm{H}_{2} \mathrm{O}_{2}$ fuels by conjugated CAT provided the propulsion force to enhance the specific binding between mannose moieties on Janus micromotors and E. coli, which initiated the AIE effect of TPE grafts and instant fluorescence "turn-on" of Janus micromotors. The PSMA side of JFRs was grafted with PEG (1), followed by TPEC grafting (2) and mannose immobilization (3). The Boc groups on the PSMA-HDA-Boc sides of the JFRs were removed after incubation with trifluoroacetic acid (4), followed by PEI grafting (5) and CAT immobilization (6). b Photographs of Janus micromotors suspensions taken under UV lamp illumination at $365 \mathrm{~nm}$ and fluorescence spectra of Janus micromotors suspensions after incubation with $E$. coli at different concentrations. Reproduced by permission of The Royal Society of Chemistry from ref. [95].

Copyright 2019 Royal Society of Chemistry

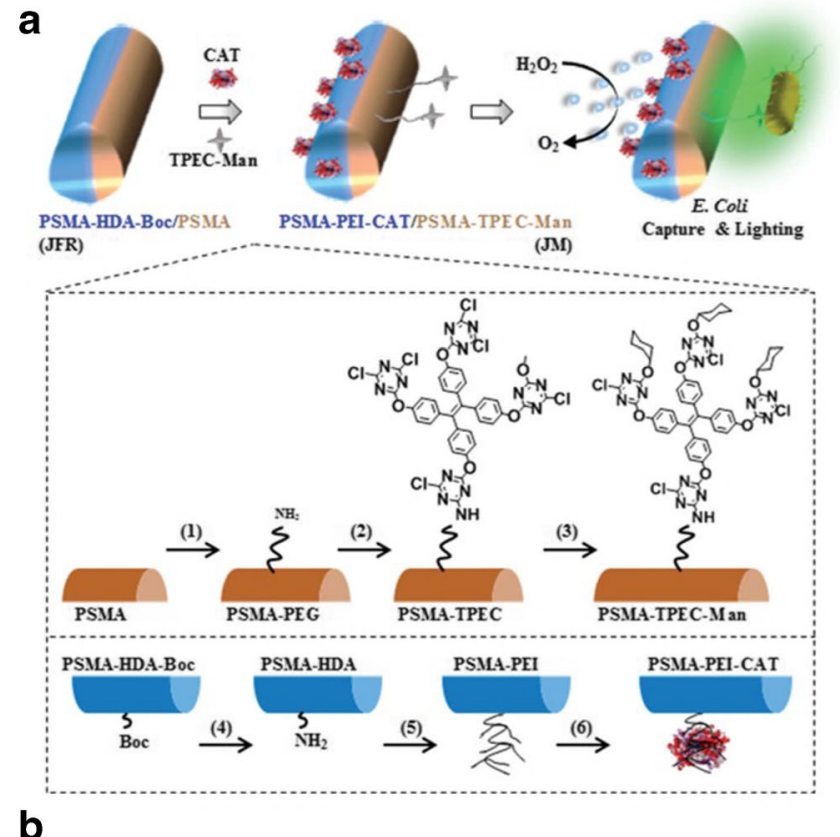

b
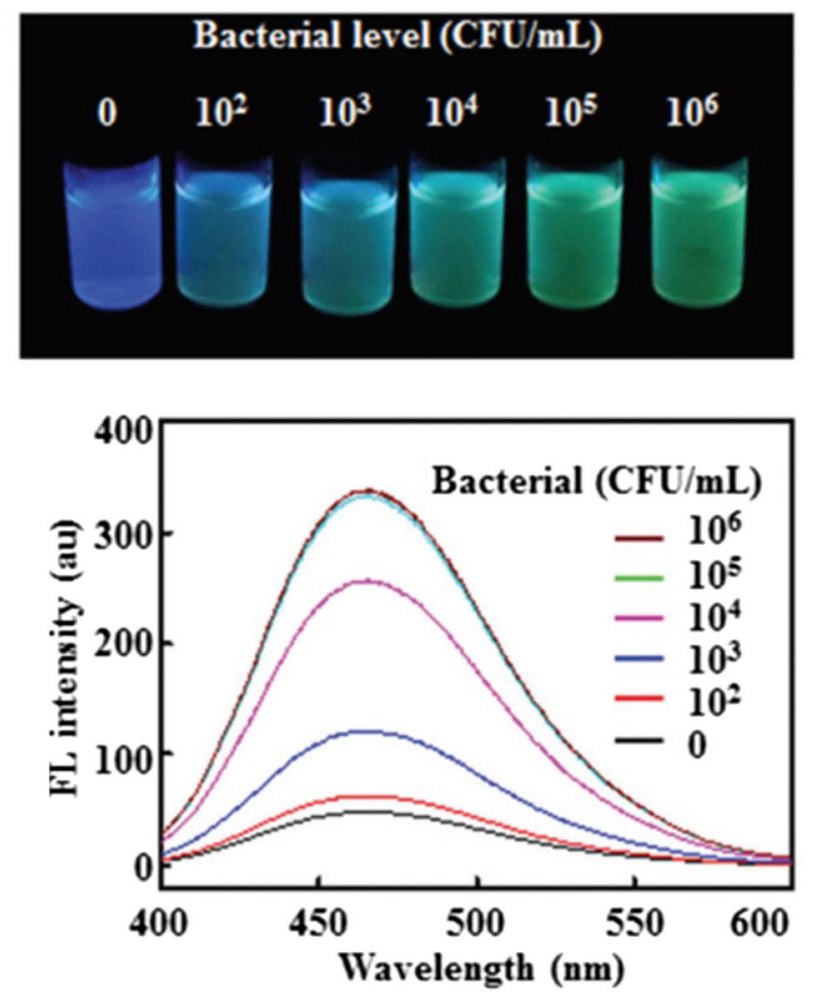

often used in this context since titania is photocatalytically active, cost-efficient, widely available, and chemically stable [99]. Li, Jia et al. demonstrated the synthesis of an array of gold-titania Janus NPs for the photocatalytic degradation of methylene blue, which is often used as model pollutant [11]. In this simple approach, monolayers of organic-inorganic hybrid films are generated by spin-coating of a mixture of polystyrene-block-poly (ethylene oxide) $/ \mathrm{HAuCl}_{4}$ solution and titania sol-gel precursor solution, where $\mathrm{HAuCl}_{4}$ and 

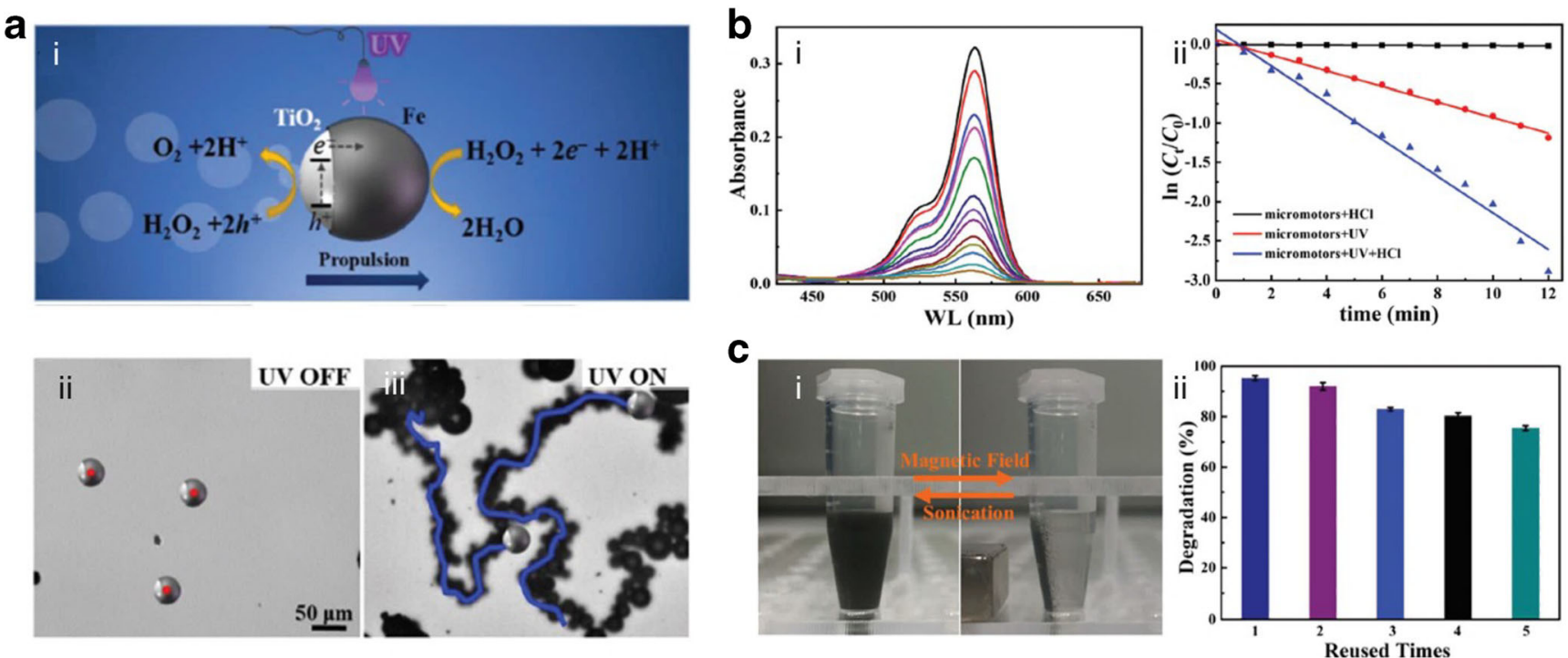

Fig. 6 a Schematic of the propulsion mechanism of the $\mathrm{TiO}_{2}-\mathrm{Fe}$ micromotors (i) and images illustrating the state of the Janus micromotors without (ii) and with UV light (iii). b Absorbance spectra of rhodamine 6G $\left(c=5 \mathrm{mg} \mathrm{mL}^{-1}\right)$ over time with micromotors with $\mathrm{UV}$ irradiation at $\mathrm{pH} 2.5,5 \% \mathrm{H}_{2} \mathrm{O}_{2}, 1 \%$ SDS in a total volume of $600 \mu \mathrm{L}$ (i) and photodegradation of rhodamine $6 \mathrm{G}$ over time with micromotors and

titania were incorporated in the PEO domains [11]. The polymer matrix was removed by deep UV irradiation and array of $\mathrm{Au}-\mathrm{TiO}_{2}$ Janus NPs were obtained [11]. The $\mathrm{Au}-\mathrm{TiO}_{2}$ heterointerfaces of the Janus NPs provided an enhanced photocatalytic activity for the degradation of methylene blue compared to bare $\mathrm{TiO}_{2}$ or $\mathrm{Au}-\mathrm{TiO}_{2}$ composite NPs [11]. In 2014, Chen, $\mathrm{Lin}$ and coworkers developed a different type of $\mathrm{TiO}_{2}$ based JPs and employed them as photocatalysts for rhodamine B degradation under UV irradiation [100]. In their synthesis approach, titanium dioxide/3-(trimethoxysilyl) propylmethacrylate (MPS) was obtained by emulsion swelling polymerization of core-shell polystyrene @ $\mathrm{TiO}_{2}$ particles [100]. The MPS lobe was then coated with a phenolic resin. After sintering in nitrogen atmosphere, porous snowmanshaped $\mathrm{TiO}_{2} /$ carbon JPs were obtained, which offered a more efficient photocatalytic degradation of rhodamine B than titanium dioxide hollow microspheres [100]. In 2016, Jassal, Agrawal et al. synthesized sub-micron sized titania-silica JPs using a Pickering emulsion strategy and calcined them afterwards [101]. The obtained silica particles half-coated with titania NPs provided a significantly higher adsorption and photocatalytic degradation rate of Solophenyl green dye compared to other titania structures [101]. The excellent photocatalytic performance was attributed to the unique Janus morphology: holes were generated in the valence band of titania, which could easily migrate to the valence band of Ti-O-Si at the interface [101]. This way, electron-hole pair separation was facilitated providing high carrier lifetimes [101]. In the same year, Guan et al. published titania-free multifunctional magnetic oleic acid-coated $\mathrm{MnFe}_{2} \mathrm{O}_{4} /$ polystyrene JPs [82].
$\mathrm{H}_{2} \mathrm{O}_{2}$ under different conditions (ii). c Photograph of the micromotors without and with external magnetic field (i); histogram of the photodegradation efficiency of rhodamine 6G after 10-min treatments with the same micromotors for five times (ii). Reproduced by permission of The Royal Society of Chemistry from ref. [106]. Copyright 2019 Royal Society of Chemistry

These JPs, which could be simply prepared on a large scale, degraded organic dyes such as rhodamine B with hydroxyl radicals generated from the Fenton reaction between $\mathrm{MnFe}_{2} \mathrm{O}_{4}$ and hydrogen peroxide [82].

Recently, Pan et al. reported on hybrid silica JPs with sheetlike shape carrying bispecific artificial receptors for simultaneous adsorption 2,6-dichlorophenol and lead(II) [69]. The Janus nanosheets were fabricated in a self-assembled sol-gel process at the oil-in-water emulsion interface with subsequent grafting of the receptors [69]. One side offered a molecularly imprinted surface for binding of 2,6-dichlorophenol, and the other side was functionalized with cysteine with affinity to lead(II) [69]. These hybrid Janus nanosheets offered fast binding kinetics $(60 \mathrm{~min})$, large monolayer adsorption capacity (129.4 $\mathrm{mg} \mathrm{g}^{-1}$ ), and high affinity to the pollutants [69].

A few years ago, research tendencies changed from static JP systems towards dynamic and self-propelling micromotors for the treatment of wastewaters. Joseph Wang and his group demonstrated a dynamic solution approach for the efficient water purification [102]. They fabricated self-propelled activated carbon-based JP micromotors that are able to move in environmental media and, thus, to remove many organic and inorganic pollutants "on-the-fly" [102]. The Janus micromotors consist of activated carbon microspheres with a microporous patch of catalytic platinum, which enables their bubble propulsion with speeds of over $500 \mu \mathrm{m} \mathrm{s}^{-1}$ [102]. The activated carbon removed nitroaromatic explosives, organophosphorus nerve agents, and azo dyes [102]. A related system was developed by Guan et al. in the same year, who showed water-fueled $\mathrm{TiO}_{2} / \mathrm{Pt}$ Janus submicromotors for "on- 


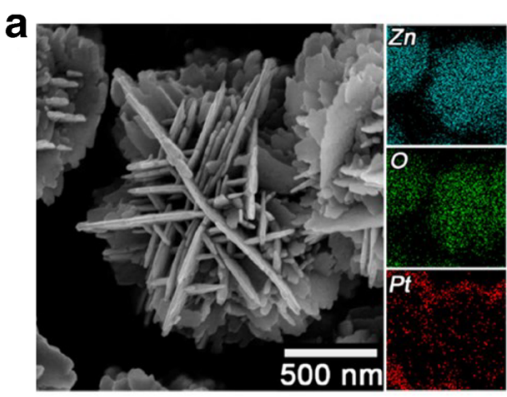

b

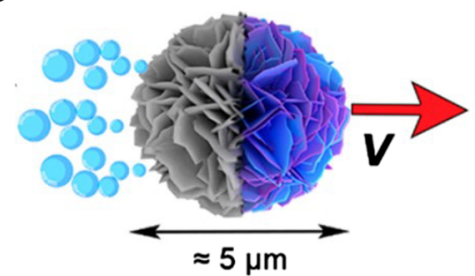

Fast-motion via bubble propulsion

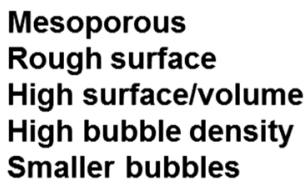

Mesoporous

Rough surface

High surface/volume

High bubble density

Smaller bubbles

C

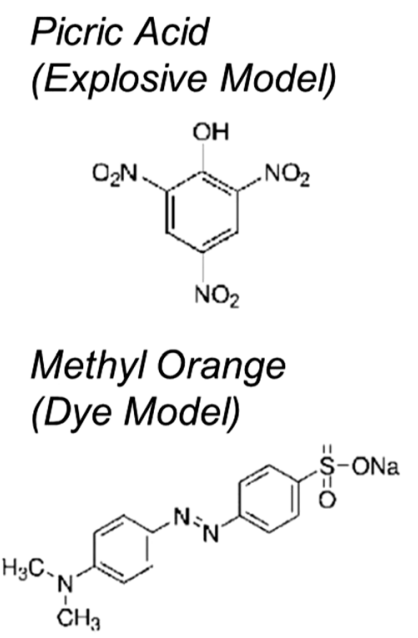

Picric Acid (Explosive Model)

\section{Methyl Orange}

Fig. 7 a Scanning electron micrographs of hierarchical and mesoporous $\mathrm{ZnO} / \mathrm{Pt}$ Janus micromotors with an average size of $1.5 \mu \mathrm{m}$ with EDS data. b Schematic of the proposed motion mechanism of $\mathrm{ZnO} / \mathrm{Pt}$ Janus

the-fly" degradation of organic dyes [103]. The motion of the JPs was governed by light-induced self-electrophoresis under the local electrical field generated by the asymmetrical water oxidation and reduction on the particle surface, and could be regulated by on/off switch, intensity and mode of the UV light [103]. A further example of dynamic Janus micromotors by Merkoçi et al. utilizes reduced graphene oxide-coated silicaPt Janus magnetic micromotors, which were used for the rapid and efficient removal of persistent organic pollutants (POPs) such as polybrominated diphenyl ethers (PBDEs) and 5chloro-2-(2,4,dichlorophenoxy) phenol (triclosan) [104]. Chang and coworkers used multifunctional zerovalent-iron/ Pt Janus bubble-propelled micromotors for the dynamic degradation of organic pollutants [105]. A patch of platinum

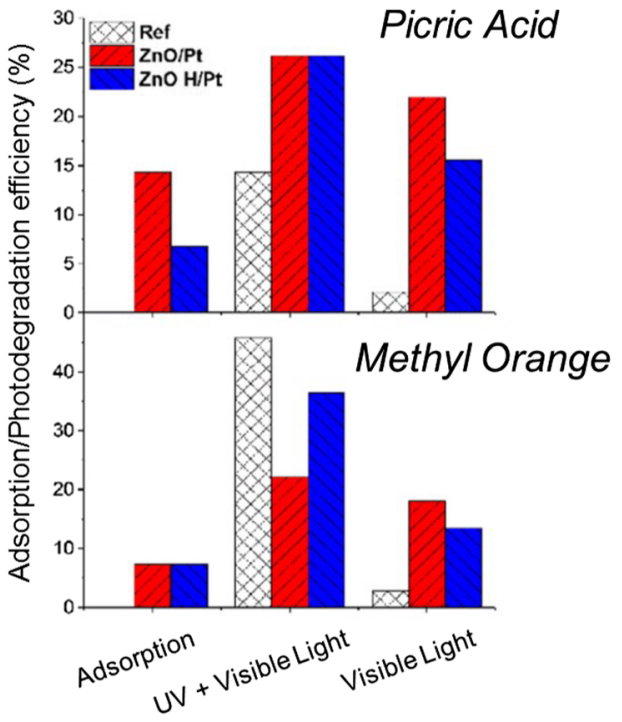

micromotors. c Adsorption and photodegradation efficiency of $\mathrm{ZnO} / \mathrm{Pt}$ micromotors. Reproduced with permission from ref. [107]. Copyright 2018 American Chemical Society

deposited onto one hemisphere of zerovalent-iron microparticles enabled the locomotion of the spheres, while the zerovalent iron acted as a heterogeneous Fenton-like catalyst [105]. The motion of the JPs enabled a much faster degradation of methylene blue compared to static iron microspheres [105]. Mei, Cai et al. enhanced this approach to novel two-inone $\mathrm{TiO}_{2}$-Fe Janus micromotors, which combined photocatalytic and Fenton reactions [106]. One hemisphere of the plain titania particles with a diameter of $15 \mu \mathrm{m}$ was coated with iron (Fig. 6a) [106]. In the presence of hydrogen peroxide and under UV irradiation, the micromotors moved due to photocatalytic decomposition of hydrogen peroxide on the titania sides (Fig. 6a) [106]. The iron-coated hemisphere induced the degradation of organic pollutants, as shown in Fig. $6 \mathrm{~b}$ for 
a
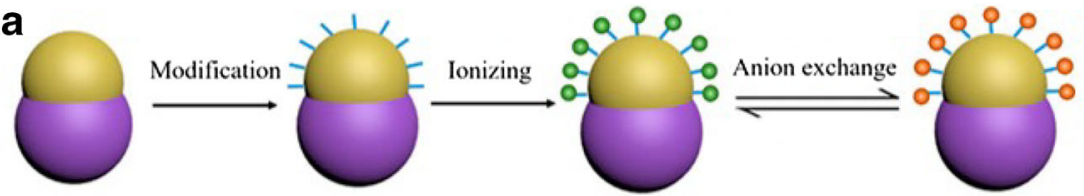

Q: Poly(3-Methacryloxypropyltrimethoxysilane) (PMPS)

: Polysty<smiles>CCO[Si](CCCN1C=NCC1)(OCC)OCC</smiles>

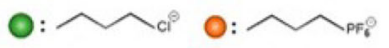
$\mathrm{CH}_{3} \mathrm{CH}_{3}$

C
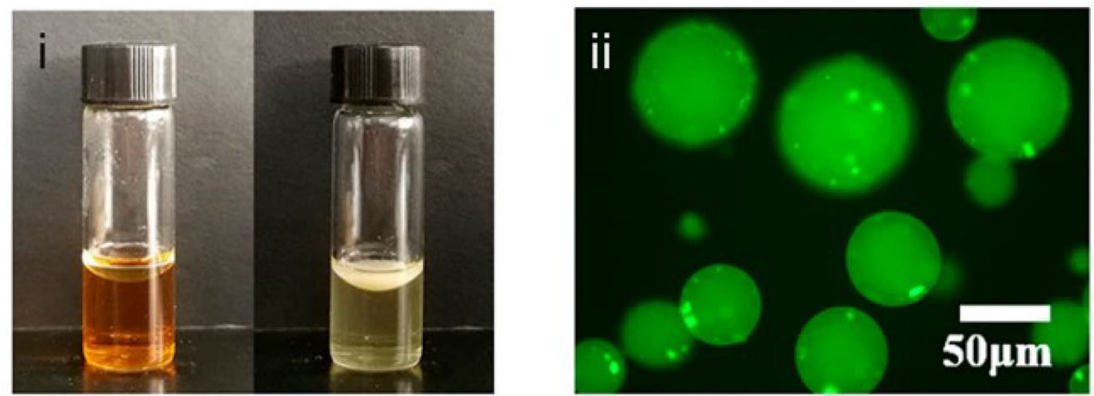

Fig. 8 a Schematic of the synthesis of JPs modified with ionic liquids and the anion exchange process. b SEM and inset TEM images of ionicliquid-silica@PDVB/PS JPs with $\mathrm{PF}_{6}{ }^{-}$as anions. c Immiscible toluenewater mixture (i, left) and emulsion after degradation of methyl orange in the presence of $15 \mu \mathrm{L} \mathrm{H} \mathrm{H}_{2}$ (i, right); optical microscopy image of the

rhodamine $6 \mathrm{~b}$, in a Fenton process [106]. The combination of photocatalytic propulsion and photo-Fenton process increased the degradation efficiency to the 52-fold of only Fenton effects and improved the bare photocatalytic performance by $40 \%$ [106]. Furthermore, the micromotors could be easily separated by an external magnet due to the magnetic properties of the iron and reused for at least five times with still high degradation efficiencies of 75\% layer (Fig. 6c) [106].

The spectrum of chemical functionalities for micromotors was further broadened by the group of Martin Pumera [107]. They produced mesoporous spherical $\mathrm{ZnO} / \mathrm{Pt}$ Janus micromotors for the efficient removal of nitroaromatic explosives and dye pollutants by photodegradation under visible light (Fig. 7) [107]. In a first step, porous flower-shaped $\mathrm{ZnO}$ particles were produced based on nanosheet assemblies, onto which the catalytic platinum layer was deposited afterwards in a physical vapor deposition process (Fig. 7a) [107]. The mesoporous $5 \mu \mathrm{m} \mathrm{ZnO/Pt} \mathrm{Janus} \mathrm{micromotors} \mathrm{move} \mathrm{via}$ catalytic bubble propulsion in the presence of $\mathrm{H}_{2} \mathrm{O}_{2}$ (Fig. 7b) [107]. The water purification capability of the mesoporous micromotors was demonstrated using two model pollutants, picric acid as explosive model and methyl orange as dye model, by both adsorption and photodegradation (Fig. 7c). The adsorption and photodegradation capability of picric acid and methyl orange was three times higher for porous $\mathrm{ZnO} / \mathrm{Pt}$ micromotors compared to nonporous $\mathrm{ZnO}-\mathrm{H} / \mathrm{Pt}$ particles

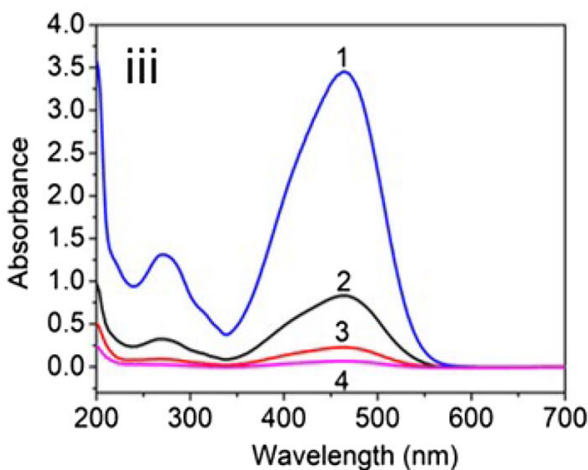

b

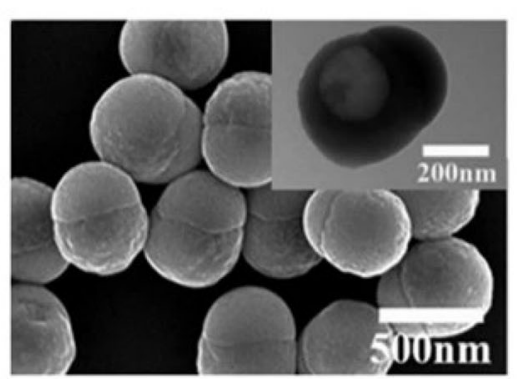

Wavelength $(\mathrm{nm})$

toluene-in-water emulsion (ii); UV-vis spectra of methyl orange (50 ppm, aqueous, curve 1), after degradation with JPs with $\mathrm{PW}_{12} \mathrm{O}_{40}{ }^{3-}$ as anions in water phase without $\mathrm{H}_{2} \mathrm{O}_{2}$ (curve 2), with $\mathrm{H}_{2} \mathrm{O}_{2}$ (curve 3) and in oil-inwater emulsion with $\mathrm{H}_{2} \mathrm{O}_{2}$ (curve 4) (iii). Reproduced with permission from ref. [12]. Copyright 2019 American Chemical Society

since they have a larger active surface area and faster motion (Fig. 7c) [107].

Enhancements of self-propelled inorganic Janus micromotors were shown by Guan, Pumera et al. [108]. They generated a 3D-printed millimeter-scale motor acting as "aircraft carrier" of $\mathrm{TiO}_{2} / \mathrm{Pt}$ Janus micromotors [108]. The carrier motor could carry and release the Janus micromotors, which can be then propelled by light illumination while acting as photodegradation agents [108]. The 3D-printed motors moved fast for tens of meters due to the Marangoni effect by asymmetrically releasing ethanol [108]. Nitroaromatic explosives could be efficiently degraded over a large solution area [108].

However, the degradation of organic pollutants by JPs is not limited to purely inorganic systems. The introduction of organic matter to create hybrid JP systems broadens the spectrum of possibilities in synthesis and application. In 2019, an organic-inorganic hybrid micromotor was presented by Guo et al. [83]. Combining the means of microfluidics and phase separation, monodisperse bubble-propelled micromotors with controllable sizes and shapes could be obtained in large quantities [83]. The incorporation of $\mathrm{MnO}_{2}$ and $\mathrm{Fe}_{3} \mathrm{O}_{4} \mathrm{NPs}$ into the ethoxylated trimethylolpropane triacrylate matrix enabled bubble production from hydrogen peroxide and catalytic pollutant degradation, respectively [83]. Thus, methylene blue as model pollutant could be effectively degraded up to $93.8 \%$ within $4.5 \mathrm{~h}$, which is significantly improved compared to 
a

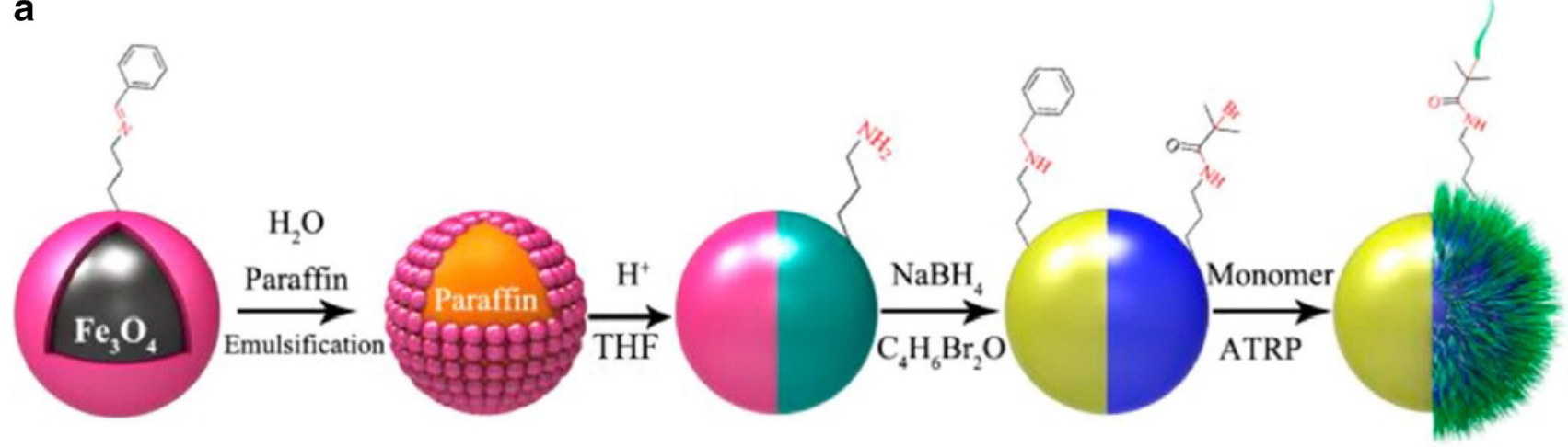

: Phenyl modified $\mathrm{Fe}_{3} \mathrm{O}_{4} \mathrm{aSiO}_{2}$ - PIL

b
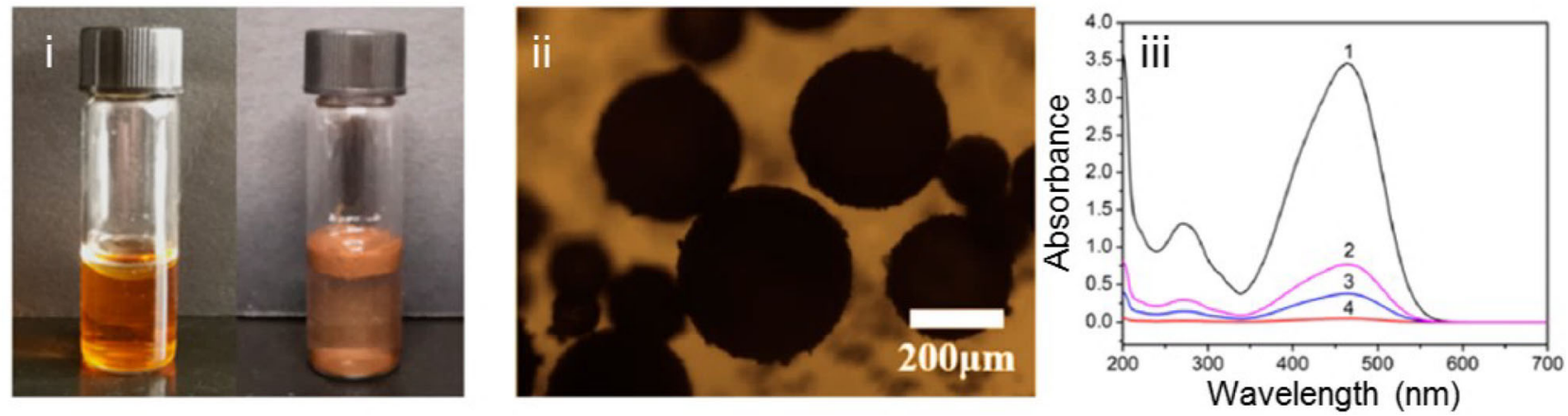

Fig. 9 a Schematic of the synthesis of magnetic JPs modified with poly(ionic liquid). b Immiscible toluene-water mixture (i, left) and emulsion after degradation of methyl orange in the presence of $20 \mu \mathrm{L} \mathrm{H}_{2} \mathrm{O}_{2}$ (i, right); optical microscopy image of the toluene-in-water emulsion (ii); UV-vis spectra of methyl orange (50 ppm, aqueous, curve 1 ), after degradation with JPs with $\mathrm{PW}_{12} \mathrm{O}_{40}{ }^{3-}$ as anions in water phase without $\mathrm{H}_{2} \mathrm{O}_{2}$ (curve 2), with $\mathrm{H}_{2} \mathrm{O}_{2}$ (curve 3) and in oil-in-water emulsion with $\mathrm{H}_{2} \mathrm{O}_{2}$ after $8 \mathrm{~h}$ (curve 4) (iii). Reproduced with permission from ref. [84]. Copyright 2019 American Chemical Society

hairy JPs were then used as solid emulsifiers (Fig. 9b), which provide catalytic properties for the degradation of watersoluble dyes such as methyl orange [84].

\section{Removal and recovery of oil}

At the moment, fossil fuels, i.e., mineral oil, ensure a large part of our living standard despite a lot of environmental and political drawbacks going hand in hand with the use of mineral oil as energy and material source. Commonly used flooding techniques with surfactants, polymers, and/or alkali are more and more restricted due to severe environmental pollution, harsh reservoir condition but also high chemical costs. Thus, nanofluid flooding has attracted increasing attention since it is low-cost and a more environmentally friendly enhanced oil recovery technique. Silicon- and metal oxide-based NPs are conventionally used for nanofluid flooding, but they suffer from low efficiencies due to their missing amphiphilicity. JPs offer great potential for an amphiphilic material design, which can be employed the oil recovery processes. 

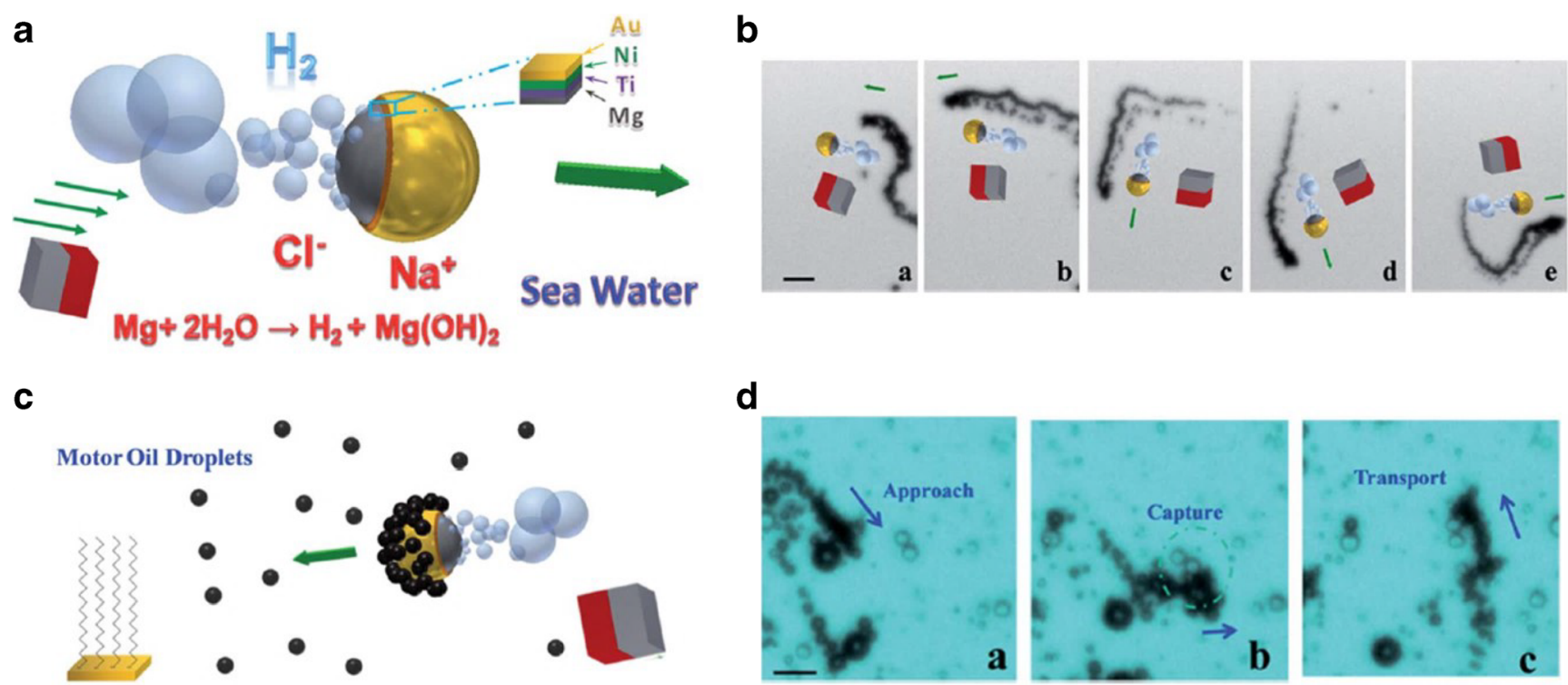

Fig. 10 (a) Schematic of the Mg-based seawater-driven Janus micromotors. (b) Time-lapse images showing the propulsion of a micromotor in seawater in 1-s intervals with spatial magnetic motion control. Scale bar: $40 \mu \mathrm{m}$. (c) Schematic hydrophobic seawater-driven alkanethiol-modified Mg-based micromotors for environmental oil

remediation. (d) Time-lapse images of Mg-based Janus micromotors with approach, capture, and transport of the oil droplet in seawater. Scale bar: $50 \mu \mathrm{m}$. Reproduced by permission of The Royal Society of Chemistry from ref. [42]. Copyright 2013 Royal Society of Chemistry

Ren et al. demonstrated a nanofluid containing a low concentration $(0.01 \mathrm{wt} \%)$ of graphene-based amphiphilic nanosheets for tertiary or enhanced oil recovery [109]. These nanosheets accumulate at the oil-water interface and reduce the interfacial tension in a saline medium [109]. A climbing solid-like interfacial film was formed and the oil phase was rapidly separated from the water phase with an efficiency of about $15.2 \%$ [109]. Such sheet-like Janus structures benefit from their rotation restriction, leading to more stable emulsions, and a higher surface-to-volume ratio compared to spherical counterparts. In a following contribution by the same group, the graphene-based amphiphilic Janus nanosheets were applied for secondary oil recovery to eliminate the tertiary stage and save large amounts of water [110]. The Janus nanosheets provided an increased oil recovery by up to $7.5 \%$ in a saline environment [110]. Although the efficiency is lower compared to the former publication, this approach benefits from lower concentrations of nanosheets, huge amounts of saved water, and higher oil production rates for fast cost recovery [110].

Recently, Yang et al. reported on carboxy/alkyl composite silica-based amphiphilic Janus nanosheets for tertiary or enhanced oil recovery [111]. Due to the high interfacial activity of the Janus nanosheets, the efficiency of oil recovery could be increased to $18.31 \%$ even at ultralow nanosheet concentrations of $0.005 \mathrm{wt} \%$ [111].

On the other hand, millions of tons of spilled oil are released by accident to the aquatic environment each year having severe consequences for the ecological system. Therefore, the removal of oil spill from contaminated seawater is of high

importance to minimize environmental hazards. However, many common cleaning materials including clay, activated carbon, and natural fibrous sorbents are neither efficient nor selective due to their weak affinity to oil [82]. Filtration systems utilizing porous membranes are another option for the removal of oil, but they are susceptible to membrane fouling and are also energy-consuming [82]. Hence, it is indispensable to develop multifunctional materials for economic and ecologic oil removal from polluted water.

The group of Yang et al. was one of the first, who employed amphiphilic Janus nanosheets as solid surfactants to collect oil spills [112]. The large-scale synthesis of the Janus nanosheets was carried out by crushing silica Janus hollow spheres [112]. To impose a magnetic response, $\mathrm{Fe}_{3} \mathrm{O}_{4}$ NPs were adsorbed onto the amino-terminated side of the Janus nanosheets [112]. The obtained Janus nanosheets successfully stabilized oil droplets in water, which could be then collected by an external magnet [112]. Another example was given by Guan et al., who showed oleic acid-coated $\mathrm{MnFe}_{2} \mathrm{O}_{4} /$ polystyrene JPs simply prepared on a large scale via phase separation [82]. These JPs offer three meaningful properties: amphiphilicity, magnetic properties, and catalytic activity [82]. The amphiphilicity of the JPs enabled encapsulation of oil droplets by the JPs. Afterwards, the encapsulated oil droplets could be separated by an external magnetic field within $2 \mathrm{~min}$ and removed from the water [82]. A further interesting approach for the separation of oil was published by Wang et al. in 2018 [113]. They reported on magnetic JPs with a convex hydrophilic and concave oleophobic surface synthesized via emulsion interfacial polymerization and selective 


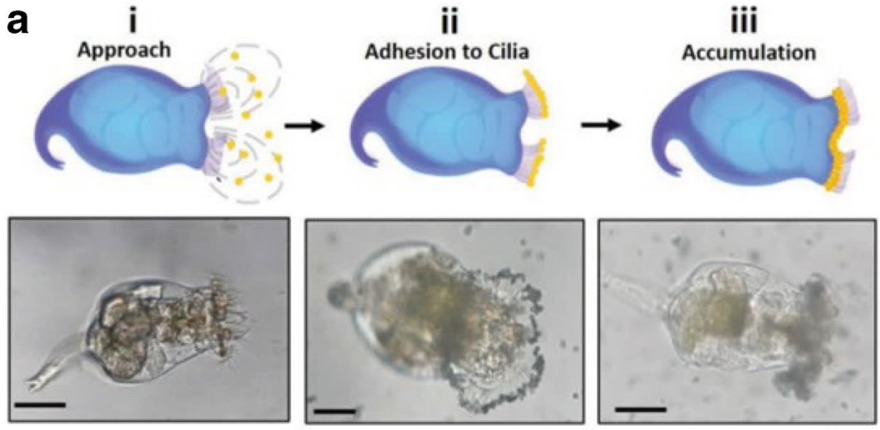

C
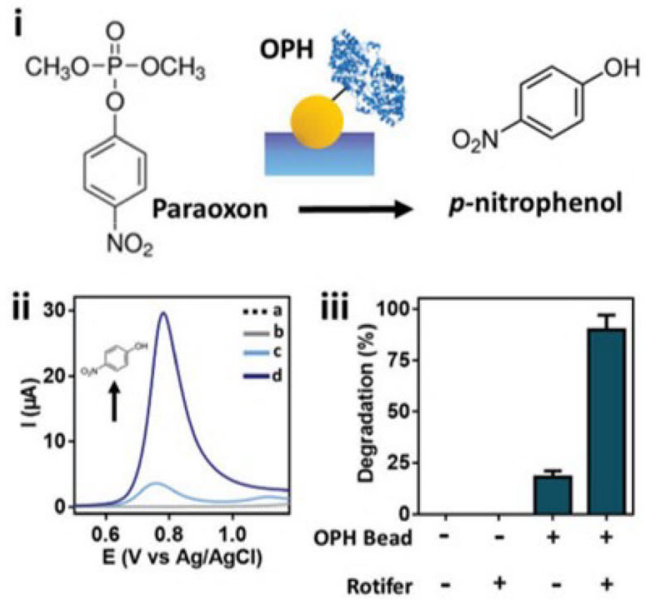

Fig. 11 a Schematic and microscopy images of the mechanism for the formation of the rotibot (scale bar: $50 \mu \mathrm{m}$ ). b Lysozyme-modified rotibotbased antibacterial treatment: schematic illustration of lysozyme microbeads for killing of $E$. coli bacteria; SEM micrographs showing live and dead $E$. coli before and after the treatment with lysozyme-modified rotifers, respectively (scale bar: $2 \mu \mathrm{m}$ ); statistical plot showing the $E$. coli bacteria killing efficiency under different treatment conditions. c Schematic illustrating the degradation of the nerve agent methyl paraoxon by OPH-microbead-modified rotibots (i); square wave voltammetry for

surface assembly [113]. These magnetic JPs were able to separate tiny microscaled oil droplets from water within $120 \mathrm{~s}$ and an efficiency of more than 99\% [113]. Micro-sized oil droplets coalesce into larger oil droplets in the presence of the JPs, which act as solid surfactants to stabilize the large droplets [113]. Finally, by employing an external magnet, the oil droplets can be easily removed from the water phase [113].

In 2013, Joseph Wang and coworkers demonstrated a more dynamic approach using hydrogen bubble-propelled Janus micromotors [42]. The locomotion is based on the magnesiumwater reaction and, thus, the JPs propel autonomously in seawater without the need for any external fuel [42]. These Janus micromotors consist of biodegradable, environmentally friendly, and low-cost $30-\mu \mathrm{m}$ magnesium microparticles that have a nickel-gold patch for magnetic guidance and further surface functionalization [42]. The propulsion mechanism combined macrogalvanic corrosion and pitting corrosion effects in chloride-rich environments, such as seawater [42]. The gold b

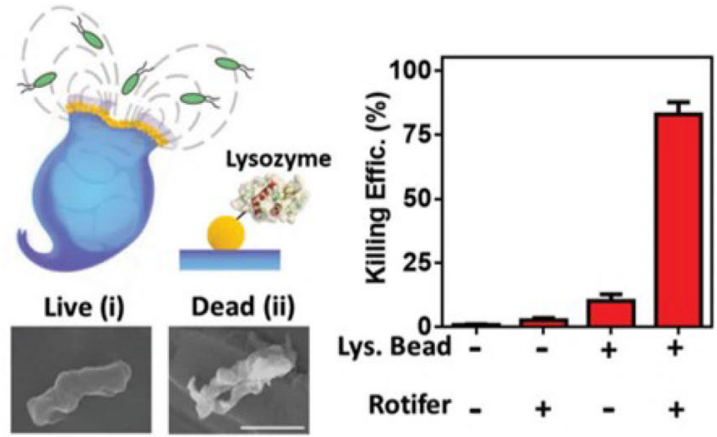

d
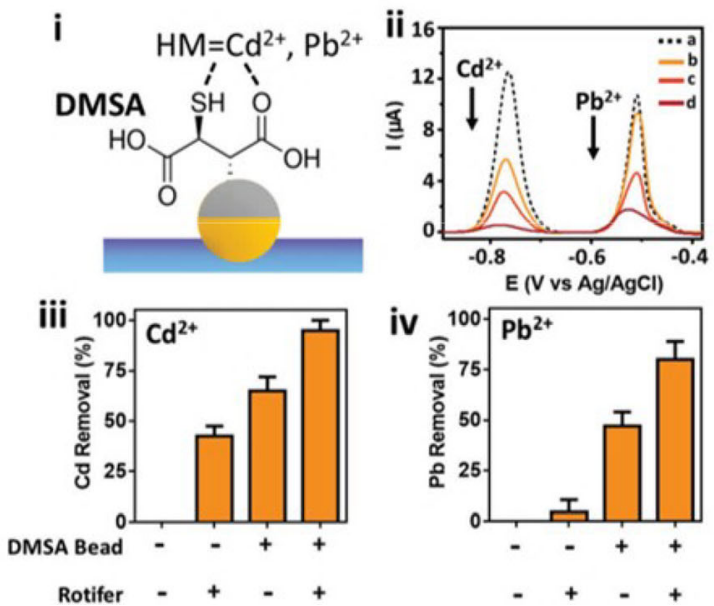

decontamination after negative control. Bare rotifer, OPH-microbeads and OPH-modified rotibot (ii); statistical plot showing the degradation efficiency under different treatments (iii). d Schematic illustrating chelation of heavy metals by DMSA-Janus microbead-modified rotibots (i); square wave voltammetry for $\mathrm{Cd}^{2+}$ and $\mathrm{Pb}^{2+}$ presence after negative control, bare rotifers, DMSA microbeads, and DMSA-modified rotibot (ii); statistical plot showing the removal efficiency of $\mathrm{Cd}^{2+}$ (iii) and $\mathrm{Pb}^{2+}$ (iv) by different treatments. Reproduced with permission from ref. [114]. Copyright 2019 WILEY-VCH Verlag GmbH \& Co- KGaA, Weinheim

patch was modified with long-chain alkanethiols leading to a super-hydrophobic surface which could be used for environmental "on-the-fly" oil remediation. The Janus micromotors were guided by an external magnet to approach, captured oil droplets, and transported them with a speed of $44 \mu \mathrm{m} \mathrm{s}^{-1}$ [42].

\section{Removal of metals}

Heavy metal ions, such as $\mathrm{Pb}^{2+}$ or $\mathrm{Ag}^{+}$, can be found in industrial sewage, posing a serious threat to public health and environment due to their high toxicity. Thus, water decontamination and environmental remediation are indispensable to ensure an acceptable water quality.

In 2015, the group of Joseph Wang demonstrated selfpropelled activated carbon-based JP micromotors for efficient and rapid "on-the-fly" removal of heavy metals, but also nitroaromatic explosives, organophosphorus nerve agents, and azo dyes [102]. The asymmetric deposition of a catalytic 

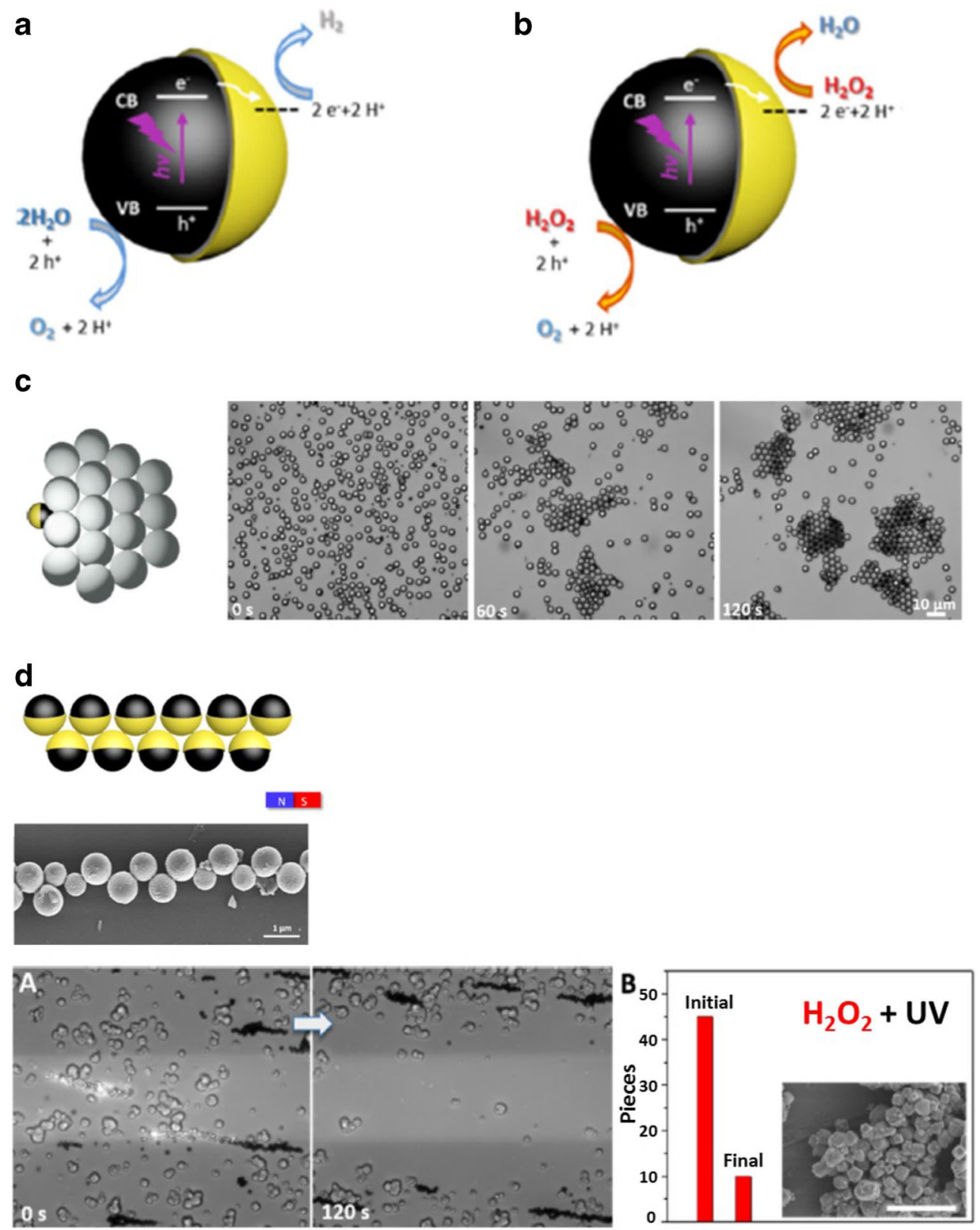

Fig. 12 Schematic of the photocatalytic propulsion of $\mathrm{Au} @ \mathrm{Ni} @ \mathrm{TiO}_{2}$ micromotors in water (a) and in $0.1 \% \mathrm{H}_{2} \mathrm{O}_{2}$ solution under UV light $(315 \mathrm{~mW})(\mathbf{b})$. c Scheme of the phoretic interaction between $\mathrm{Au} @ \mathrm{Ni} @ \mathrm{TiO}_{2}$ micromotors and PS particles; time-lapse optical images illustrating Au@Ni@ $\mathrm{TiO}_{2}$ micromotors, collecting PS particles by phoretic interaction in $1.67 \% \mathrm{H}_{2} \mathrm{O}_{2}$ under UV light $(315 \mathrm{~mW})$. d Scheme and SEM image of chain formation of magnetic

platinum patch onto the rough surface of activated carbon microspheres allows the bubble propulsion of the Janus micromotors with high speeds of more than $500 \mu^{-1}$ s song with high adsorption capacity of $\mathrm{Pb}^{2+}[102]$. In a following contribution from the same group, a different type of selfpropelled microrobots, employing marine rotifers, was presented [114]. These biohybrid so-called rotibots were fabricated via electrostatic adsorption of functionalized positively
$\mathrm{Au} @ \mathrm{Ni} @ \mathrm{TiO}_{2}$ micromotors under magnetic field; magnetic $\mathrm{Au} @ \mathrm{Ni} @ \mathrm{TiO}_{2}$ chains remove microplastics from washing powder in $0.1 \% \mathrm{H}_{2} \mathrm{O}_{2}$ under magnetic field and 63-mW UV light; amount of microplastic particles before and after removal; insets show SEM images. Scale bar: $10 \mu \mathrm{m}$. Reproduced with permission from ref. [117]. Copyright 2019 American Chemical Society

charged microbeads on the negatively charged surfaces of the rotifers' cilia (Fig. 11a) [114]. The high fluid flow towards the mouth led to a very efficient transport of contaminated water to the functionalized microbeads [114]. To chelate and remove heavy metal ions, such as $\mathrm{Cd}^{2+}$ or $\mathrm{Pb}^{2+}$, the microbeads were modified with the ligand meso-2,3dimercaptosuccinic acid (DMSA) (Fig. 11d) [114]. Further environmental remediation applications were achieved by 
a

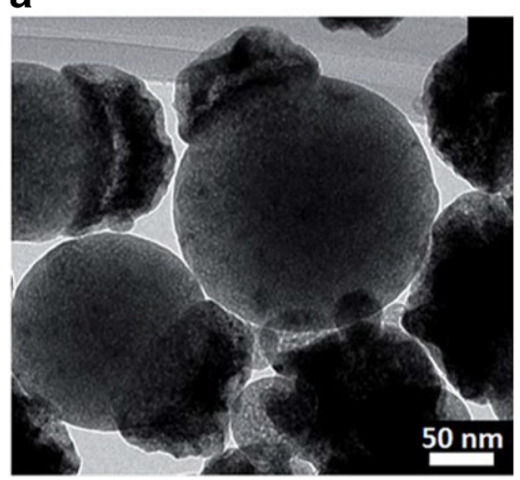

b

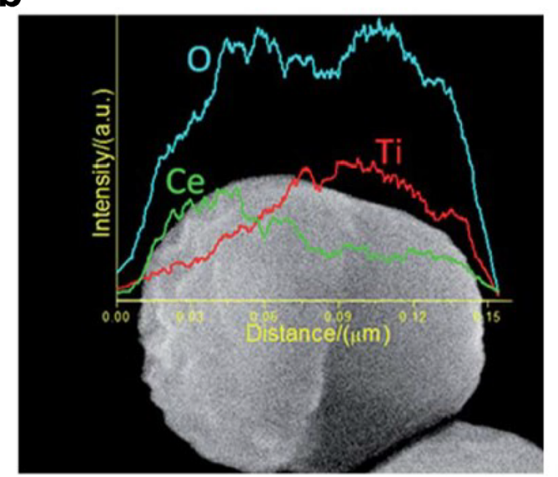

C

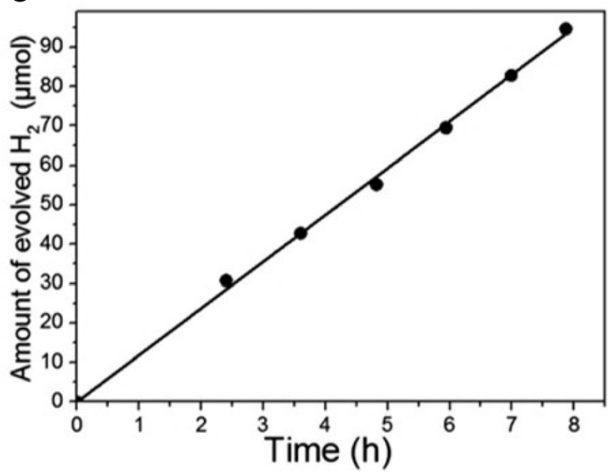

Fig. 13 a TEM image of snowman-like $\mathrm{CeO}_{2}-\mathrm{TiO}_{2}$ JPs. b Linear EDX analysis of $\mathrm{Ce}, \mathrm{Ti}$, and $\mathrm{O}$ elements over a typical JP. c Time-dependent hydrogen evolution from water with snowman-like $\mathrm{CeO}_{2}-\mathrm{TiO}_{2}$ JPs and
$\mathrm{Na}_{2} \mathrm{~S}(0.1 \mathrm{M})$ and $\mathrm{Na}_{2} \mathrm{SO}_{3}(0.02 \mathrm{M})$ under the irradiation of simulated sunlight. Reproduced by permission of The Royal Society of Chemistry from ref. [13]. Copyright 2013 Royal Society of Chemistry functionalization of the microcleaners with lysozyme and organophosphorus hydrolase for enzymatic biodegradation of Escherichia coli (Fig. 11b) and the nerve agent methyl paraoxon (Fig. 11c), respectively [114].

Another example of self-propelled micromotors for removal of metals was demonstrated by Park et al., who reported on magnetically steerable mesoporous silica microspheres functionalized with copper ferrocyanide, ferromagnetic nickel, and catalytic platinum layers [115]. Copper ferrocyanide is highly selective to Cs [115]. In the presence of hydrogen peroxide, the micromotors propelled via Pt-catalyzed bubble evolution, which led to an eightfold higher Cs removal of the micromotors compared to non-moving JPs [115]. Furthermore, the nickel layer made the micromotors remotely steerable and enhanced the propulsion speed to the 11-fold [115]. Thus, radioactive ${ }^{137} \mathrm{Cs}$ could be successfully adsorbed by more than $98 \%$ even in the presence of competing sodium ions [115].

\section{Removal of bacteria}

Monitoring and removal of pathogenic bacteria is a longstanding issue in environmental engineering, healthcare, and nutritional industry due to their major threats to human health especially in times of increasing resistances against antibiotics. It is estimated that about 300 million people worldwide contract a bacterial infection each year [95]. Furthermore, the exposure to pathogen-contaminated water is one of the major causes of death in many developing countries [95]. To control potential human poisoning and to prevent the spread of infections, rapid and early detection and removal of bacteria is essential. In 2012, the group of Joseph Wang demonstrated self-propelled gold/nickel/polyaniline/Pt microtubular engines, which were functionalized with the lectin bioreceptors for the rapid isolation of pathogenic Escherichia coli in real time [93]. The Janus microtubular motors were fabricated via template-based electrodeposition of a polyaniline/Pt bilayer microtube and e-beam vapor deposition of outer $\mathrm{Ni} / \mathrm{Au}$ layers. The Ni/Au layers were subsequently functionalized with alkanethiols and the lectin receptor (ConA) [93]. Apart from specific binding of the ConA-modified microengines to E. coli, the captured bacteria can be also released on demand triggered by using a low-pH glycine solution that is able to dissociate the lectin-bacteria complex [93].

An approach towards non-propelling JPs for the isolation of bacteria was proposed by Maas and coworkers, who designed nanosized bifunctional magnetite@ $\mathrm{SiO}_{2}$ Janus nanoparticles with antibodies against one particular bacterial species on one hemisphere and polyethyleneglycol (PEG) chains on the other side to prevent particle agglomeration [116]. The magnetic JPs efficiently captured E.coli bacteria from a mixture of bacteria via specific antigen-antibody interactions without compromising the viability of the captured bacteria, and could be subsequently magnetically separated [116]. The Janus character of the particles prevented the agglomeration between the bacteria, contrarily to isotropically functionalized nanoparticles [116]. The presented approach allows rapid and agglomeration-free separation of live bacteria for biotechnological purposes [116].

\section{Removal of microplastics}

Microplastic contamination has attracted attention in science and society since its remarkable persistence and harmfulness for marine organisms. Recently, Simmchen et al. developed photocatalytic Au@Ni@TiO 2 -based micromotors (Fig. 12a,b) for the removal of microplastics from personal care products and open waters [117]. They proposed two different strategies: In the first approach, individual micromotors enabled the removal based on phoretic interactions (Fig. 12c), while chain-like assemblies of these micromotors caused shoveling and pushing interactions in the second strategy (Fig. 12d). The chained assemblies work independent of the 
fuel, in diluted peroxide solution and water, which is of high importance for the practical applicability.

\section{Hydrogen production and water splitting}

Another aspect to achieve a more sustainable world is the transition from fossil fuel energy sources towards renewable energy conversion concepts, e.g., solar cells or photocatalytic hydrogen production via water splitting for the production of hydrogen. Hydrogen is a promising energy carrier due to its high energy density and the formation of clean combustion products. However, there are several challenges for the fabrication of a suitable photocatalyst, such as utilization of broadband light, efficient separation and transfer of electron-hole pairs, adequate band-edge potentials for overall water splitting, and low material costs [118]. Traditional semiconducting photocatalysts often suffer from some limitations, i.e., low quantum efficiency, charge recombination, and back-reactions, which cannot be overcome by a single material [119]. Due to the unique heterostructure with combinations of materials and anisotropic surface properties of JPs, they have gained increasing interest in photocatalytic hydrogen production [13, 118, 120-124]. In 2013, Guan and coworkers demonstrated a strategy for large-scale fabricated JPs with controlled structure and chemical composition by an oppositely charged twin-head electrospray [13]. By electrospraying two different droplets from two tip-to-tip nozzles at high voltages of opposite polarities and after solvent evaporation and precursor gelation, these droplets collided and coagulated into JPs because of attractive Coulomb forces [13]. Employing this approach, they were able to show metal oxide-metal oxide and metal oxide-metal sulfide JPs of snowman-like, hollow-bowl snowman-like, and pot-like shape [13]. As a proof of concept, snowman-like $\mathrm{CeO}_{2}-\mathrm{TiO}_{2}$ JPs were exemplarily used for photo-assisted water splitting for hydrogen production (Fig. 13a,b). The observed hydrogen generation rate reached $1185 \mu \mathrm{mol} \mathrm{h}^{-1} \mathrm{~g}^{-1}$ (Fig. 13c), which is at least ten times higher than for a $\mathrm{CeO}_{2}-\mathrm{TiO}_{2}$ composite $\left(28-102 \mu \mathrm{mol} \mathrm{h}^{-1} \mathrm{~g}^{-1}\right)[13$, 125]. The superior activity of the JPs was attributed to the presence of abundant heterojunctions, which led to efficient separation of the photogenerated electrons and holes [13].

Another example of employing JPs for hydrogen production was proposed by Liu, Mlayah, Han et al. [126]. They developed non-centrosymmetric Janus $\mathrm{Au}-\mathrm{TiO}_{2}$ particles with interesting localized-surface-plasmon-resonance (LSPR) properties and employed them as photocatalysts for efficient plasmon-enhanced visible-light hydrogen generation [126]. The JPs provided a higher rate of hydrogen generation compared to symmetric core-shell $\mathrm{Au}-\mathrm{TiO}_{2} \mathrm{NPs}$ due to stronger localization of plasmonic near-fields close to the $\mathrm{Au}-\mathrm{TiO}_{2}$ interface on one side of the Au NPs [126]. Thus, the plasmonic near-fields were strongly coupled to optical transitions involving the localized stated in amorphous titania, which caused efficient optical absorption and generation of electron-hole pairs for photocatalysis [126]. Even higher photocatalytic activity was found for increasing Au NP size up to $70 \mathrm{~nm}$ due to their stronger plasmonic near-fields [126].

A related approach was developed by Claverie et al., who demonstrated the impact of whispering gallery mode (WGM) resonances on plasmonic photocatalysis [120]. These JPs were constituted of one gold multimer $(\sim 60 \mathrm{~nm})$, which was partially embedded in a $\mathrm{TiO}_{2}$ sphere $(\sim 440 \mathrm{~nm})$ during the hydrolysis of the $\mathrm{TiO}_{2}$ precursor [120]. This Janus system as well as several other homo- and heterogeneous particle hybrids were tested as photocatalysts for water splitting under visible-NIR illumination at $\lambda>420 \mathrm{~nm}$ [120]. While pure $\mathrm{Au}$ $\mathrm{NPs}, \mathrm{TiO}_{2}$ spheres, and $\mathrm{Au} @ \mathrm{TiO}_{2}$ did not show any photocatalytic activity, the Janus Au multimer- $\mathrm{TiO}_{2}$ particles provided an even 2-4 times higher activity compared to $\mathrm{Au}$ mono- $\mathrm{TiO}_{2}$ of the same specific surface, size, and amount of Au NPs [120]. Furthermore, photoelectrochemical measurements proved that the Janus Au multimer- $\mathrm{TiO}_{2}$ system generated a photocurrent under full solar light that is increased by almost a factor of 3 compared to pure $\mathrm{TiO}_{2}$ spheres [120]. In 2017, Piao et al. showed a similar system of non-centrosymmetric $\mathrm{Au} /$ $\mathrm{TiO}_{2}$ nano-mushrooms, for which $\mathrm{TiO}_{2} \mathrm{NPs}$ are anchored at only one end of gold nanorods [122]. The hydrogen generation rate reached up to $52.6 \mu \mathrm{mol} \mathrm{g}{ }^{-1} \mathrm{~h}^{-1}$ under sunlight irradiation from pure water without assistance of electron donors [122]. It was assumed that the dominant electron transfer occurred from the $\mathrm{TiO}_{2} \mathrm{NPs}$ to the gold nanorods [122]. The increased photocatalytic efficiency was caused by the directed charge separation and the formation of spatially divided redox reaction areas [122].

JP-mediated photocatalytic hydrogen generation is not limited to single-phase media. The group of Chen fabricated anatase $\mathrm{TiO}_{2-}$ based Janus catalysts via selective surface modification for realization of catalytic hydrogen productions in biphasic systems [123]. Amphiphilic $\mathrm{TiO}_{2}$ particles grafted with 3-(trimethoxysilyl) propyl methacrylate were employed for the stabilization of a water $/ \mathrm{n}$ octanol (Pickering) emulsion with simultaneous photocatalytic hydrogen generation [123]. The $\mathrm{TiO}_{2}$-based JPs showed a much higher catalytic activity compared to bare $\mathrm{TiO}_{2}$ and to conventional biphasic systems due to the excellent emulsification properties of the JPs enabling a high accessibility for the reactants [123].

In a following contribution from the same group, amphiphilic raspberry-like $\mathrm{Ag}_{2} \mathrm{O}-\mathrm{TiO}_{2} / \mathrm{SiO}_{2}$ Janus photocatalysts were presented to enhance the efficiency of photoreforming hydrogen production in biphasic systems [124]. These JPs successfully stabilized water/octanol emulsions and the hydrogen yield was significantly increased compared to bare $\mathrm{TiO}_{2}, \mathrm{Ag}_{2} \mathrm{O}-\mathrm{TiO}_{2}$, and $\mathrm{TiO}_{2} / \mathrm{SiO}_{2}$ [124]. Thus, amphiphilic photocatalytically active JPs open new pathways for efficient biphasic photoreactions of biomass-derived oils with the use of solvents.

To obtain a low-cost material and to avoid possible backward reactions, noble metals, which are commonly used as 
electron mediators or cocatalysts, should be replaced by more reasonable alternatives. In this context, Xiong et al. demonstrated noble-metal-free JPs consisting of $\gamma$-MnS and roxbyite $\mathrm{Cu}_{7} \mathrm{~S}_{4}$ in a cation-exchange approach [118]. This Janus system enabled photocatalytic hydrogen production rates of up to $718 \mu \mathrm{mol} \mathrm{g}^{-1} \mathrm{~h}^{-1}[118]$.

Apart from JP-based hydrogen production, solar thermal conversion utilizing gallium-doped $\mathrm{ZnO} / \mathrm{Au}$ JPs was shown by Liu and Xuan in 2017 [121]. They demonstrated full-spectrum volumetric solar thermal conversion over a thin layer of "photonic nanofluids" based on Janus NPs [121]. The asymmetry of the JPs enabled the coexistence of core resonances, shell plasmons, and core-shell resonances at different wavelengths [121]. Thus, the narrowband nature of single resonances was overcome and the solar thermal conversion efficiency could be improved by $10.8 \%$ and $154 \%$ compared to core-shell particles and pure galliumdoped $\mathrm{ZnO}$ particles, respectively [121].

\section{Future perspectives and challenges}

In this review, we have highlighted recent challenges, developments, and new perspectives of JP-based materials for environmental and biotechnological applications, ranging from using of biogenic and biocompatible materials for the JP design, their recovery and reusability aspects to their use in environmental sensing and monitoring, water decontamination, and hydrogen production. These environmental applications certainly benefit from the unique properties of Janus-structured materials, including their high surface-to-volume ratio; high interfacial activity making them efficient stabilizers of two immiscible fluids; target controlling and manipulation of their interfacial activity by external signal such as temperature, light, $\mathrm{pH}$, or ionic strength and achieving switching between stable emulsions and macro-phase separation (having a polymer coating on the surface); and recovery and recycling. Also tunable surface chemistry and functionality are very beneficial since at least bifunctional morphologies are easy to realize, which finally allow their use as carrier building blocks and attachment of various catalytically active substances (e.g., enzymes, nobel NPs) on their surface or alternatively attachment of JPs to various substrates keeping still one functionality active for further reactions. Furthermore, JPs offer great potential for the replacement of conventional (fluoro-)surfactants since they create a more permeable interface (known as "skin that can breathe" [20]) due to interstices between the particles that allow chemical exchange between the two phases. Besides, environmental applications benefit from the ability of JPs to penetrate locations inaccessible to macroscopic solution approaches. The research works highlighted in this review can be employed to open new possibilities for the design of rational and advanced (multi)functional materials.
JPs provide a broad range of possibilities for sensing and removal of manifold pollutants in water and foodstuffs. Furthermore, the depletion of fossil energy resources, increasing $\mathrm{CO}_{2}$ emission into the atmosphere, and the steadily increasing energy demand require alternative energy sources. JPs as photocatalysts for hydrogen production may contribute to a solution for this set of problems. Especially self-propelled Janus micro- and nanomotors have been evolved as a promising system for environmental applications due to their larger operating distance and resulting higher efficiencies compared to nonmotile counterparts.

However, the challenge of environmental sustainability we are facing in our times demands biogenic and biocompatible materials. Although a broad variety of strategies for the fabrication of JPs with various sizes, shapes, and functionalities was developed, there is only a limited number of JPs considering this challenging and ongoing quest up to now. To avoid undesirable spreading of JPs in the environment and to facilitate multiple uses, several strategies for the recovery and recycling of JPs have been developed.

Therefore, there are two major problems to be overcome for the future environmental applications of JPs. One problem is the toxicity of several materials used for the fabrication of JPs. Recently, $\mathrm{TiO}_{2}$ powders have been classified as carcinogen by the European Commission. The practical use of silver is also restricted due to its aquatic toxicity. Also some fuels necessary for the propulsion of Janus micromotors, such as hydrazine or hydrogen peroxide, are harmful to the environment and need to be replaced or omitted. The second problem is the cost-efficient industrial implementation of JPs. Some of the frequently used materials, such as platinum, are highly expensive. Furthermore, the scalability of the JP synthesis remains critical since many strategies are too sophisticated, and cost- and time-consuming for a large-scale production.

Ultimately, several challenges have to be accepted in future directions to eliminate the current drawbacks. Many of the commonly used materials need to be replaced by environmentally friendly or biogenic alternatives, which can be (self-)degraded into harmless products after completing a process, e.g., biodegradable functional microparticles instead of plastic microbeads, and biocatalysts (enzymes) instead of synthetic catalysts. On the other hand, the JP design should offer a sufficient durability towards reusability to decrease the amount of waste and costs accruing during the fabrication process. If there is no appropriate biogenic replacement available, potential toxicity of these JPs needs to be investigated to prevent potential adverse environmental impacts and scalable methods for the JP separation have to be devised. Furthermore, scalability of the synthesis should be realized to ensure high quantities of JPs in a precise and reproducible manner for industrial application. In this context, the number of synthesis steps needs to be reduced and purification procedures may become more efficient in order to improve 
effectiveness in time and costs, but also in terms of chemical waste reduction. The range of applications should be also broadened, since there are many unsolved problems such as the removal of antibiotics or polycyclic aromatic hydrocarbons (PAHs) from wastewaters.

Despite the strong interest and progress in the research field of JPs, we believe that the potential of multifunctional JPs as perspective materials for environmental applications is yet not fully tapped. We suggest considering both natural and fully synthetic biocompatible and biodegradable polymers for the design of JPs and with this pushing forward the development of more environmentally friendly and sustainable materials in the very near future.

Acknowledgments C.M. and A.S. acknowledge the German Research Foundation (DFG, grant no. SY 125/4-1 and SY 125/11-1), and the German Federation of Industrial Research Associations (AiF, grant no. 18195BR) for funding within the program for sponsorship by Industrial Collective Research (IGF) of the German Federal Ministry of Economic Affairs and Energy based on an enactment of the German Parliament.

Author contributions The manuscript was written through contributions of all authors. All authors have given approval to the final version of the manuscript.

Funding information Open Access funding provided by Projekt DEAL. This study received funding from the German Research Foundation (DFG, grant no. SY 125/4-1 and SY 125/11-1) and the German Federation of Industrial Research Associations (AiF, grant no. 18195BR).

Open Access This article is licensed under a Creative Commons Attribution 4.0 International License, which permits use, sharing, adaptation, distribution and reproduction in any medium or format, as long as you give appropriate credit to the original author(s) and the source, provide a link to the Creative Commons licence, and indicate if changes were made. The images or other third party material in this article are included in the article's Creative Commons licence, unless indicated otherwise in a credit line to the material. If material is not included in the article's Creative Commons licence and your intended use is not permitted by statutory regulation or exceeds the permitted use, you will need to obtain permission directly from the copyright holder. To view a copy of this licence, visit http://creativecommons.org/licenses/by/4.0/.

\section{References}

1. Web of Science. Thomson Reuters. https://apps.webofknowledge. com. Accessed October 2019201

2. Yu H, Chen M, Rice PM, Wang SX, White RL, Sun S (2005) Dumbbell-like bifunctional au-Fe3O4 nanoparticles. Nano Lett 5(2):379-382. https://doi.org/10.1021/nl047955q

3. Wang C, Xu C, Zeng H, Sun S (2009) Recent progress in syntheses and applications of dumbbell-like nanoparticles. Adv Mater 21(30):3045-3052. https://doi.org/10.1002/adma.200900320

4. Yang T, Wei L, Jing L, Liang J, Zhang X, Tang M, Monteiro MJ, Chen Y, Wang Y, Gu S, Zhao D, Yang H, Liu J, Lu GQM (2017) Dumbbell-shaped bi-component mesoporous Janus solid nanoparticles for biphasic interface catalysis. Angew Chem Int Ed 56(29): 8459-8463. https://doi.org/10.1002/anie.201701640
5. Xu C, Xie J, Ho D, Wang C, Kohler N, Walsh EG, Morgan JR, Chin YE, Sun S (2008) Au-Fe3O4 dumbbell nanoparticles as dual-functional probes. Angew Chem Int Ed 47(1):173-176. https://doi.org/10.1002/anie.200704392

6. Kang C, Honciuc A (2018) Influence of geometries on the assembly of snowman-shaped Janus nanoparticles. ACS Nano 12(4): 3741-3750. https://doi.org/10.1021/acsnano.8b00960

7. Wang X, Feng X, Ma G, Yao L, Ge M (2016) Amphiphilic Janus particles generated via a combination of diffusion-induced phase separation and magnetically driven dewetting and their synergistic self-assembly. Adv Mater 28(16):3131-3137. https://doi.org/10. 1002/adma.201506358

8. Liu Y, Hu J, Yu X, Xu X, Gao Y, Li H, Liang F (2017) Preparation of Janus-type catalysts and their catalytic performance at emulsion interface. J Colloid Interface Sci 490:357-364. https://doi.org/10. 1016/j.jcis.2016.11.053

9. Zhang Q, Zhang L, Li S, Chen X, Zhang M, Wang T, Li L, Wang C (2017) Designed synthesis of au/Fe3O4@C Janus nanoparticles for dual-modal imaging and actively targeted chemophotothermal synergistic therapy of cancer cells. Chem Eur J 23(68):17242-17248. https://doi.org/10.1002/chem.201703498

10. Schick I, Lorenz S, Gehrig D, Schilmann A-M, Bauer H, Panthöfer M, Fischer K, Strand D, Laquai F, Tremel W (2014) Multifunctional two-photon active silica-coated au@MnO Janus particles for selective dual functionalization and imaging. J Am Chem Soc 136(6):2473-2483. https://doi.org/10.1021/ja410787u

11. Fu X, Liu J, Yang H, Sun J, Li X, Zhang X, Jia Y (2011) Arrays of au-TiO2 Janus-like nanoparticles fabricated by block copolymer templates and their photocatalytic activity in the degradation of methylene blue. Mater Chem Phys 130(1):334-339. https://doi. org/10.1016/j.matchemphys.2011.06.054

12. Zhao R, Yu X, Sun D, Huang L, Liang F, Liu Z (2019) Functional Janus particles modified with ionic liquids for dye degradation. ACS Appl Nano Mater 2(4):2127-2132. https://doi.org/10.1021/ acsanm.9b00090

13. Mou F, Chen C, Guan J, Chen DR, Jing H (2013) Oppositely charged twin-head electrospray: a general strategy for building Janus particles with controlled structures. Nanoscale 5(5):20552064. https://doi.org/10.1039/c2nr33523a

14. Kirillova A, Stoychev G, Ionov L, Eichhorn KJ, Malanin M, Synytska A (2014) Platelet Janus particles with hairy polymer shells for multifunctional materials. ACS Appl Mater Interfaces 6(15):13106-13114. https://doi.org/10.1021/am502973y

15. Kirillova A, Marschelke C, Friedrichs J, Werner C, Synytska A (2016) Hybrid hairy Janus particles as building blocks for antibiofouling surfaces. ACS Appl Mater Interfaces 8(47): 32591-32603. https://doi.org/10.1021/acsami.6b10588

16. Link JR, Sailor MJ (2003) Smart dust: self-assembling, selforienting photonic crystals of porous Si. Proc Natl Acad Sci 100(19):10607-10610. https://doi.org/10.1073/pnas. 1233824100

17. Yan J, Chaudhary K, Chul Bae S, Lewis JA, Granick S (2013) Colloidal ribbons and rings from Janus magnetic rods. Nat Commun 4(1):1516-1519. https://doi.org/10.1038/ncomms2520

18. Zhao B, Zhou H, Liu C, Long Y, Yang G, Tung C-H, Song K (2016) Fabrication and directed assembly of magnetic Janus rods. New J Chem 40(8):6541-6545. https://doi.org/10.1039/ C6NJ00825A

19. Casagrande C, Fabre P, Raphaël E, Veyssié M (1989) "Janus beads": realization and behaviour at water/oil interfaces. Europhysics Letters (EPL) 9(3):251-255. https://doi.org/10. 1209/0295-5075/9/3/011

20. de Gennes P-G (1992) Soft matter (Nobel lecture). Angew Chem Int Ed Eng 31(7):842-845. https://doi.org/10.1002/anie. 199208421 
21. Yan L-T, Popp N, Ghosh S-K, Böker A (2010) Self-assembly of Janus nanoparticles in diblock copolymers. ACS Nano 4(2):913920. https://doi.org/10.1021/nn901739v

22. Zhu G, Xu Z, Yang Y, Dai X, Yan LT (2018) Hierarchical crystals formed from DNA-functionalized Janus nanoparticles. ACS Nano 12(9):9467-9475. https://doi.org/10.1021/acsnano.8b04753

23. Huang Z, Zhu G, Chen P, Hou C, Yan LT (2019) Plastic crystal-tocrystal transition of Janus particles under shear. Phys Rev Lett 122(19):198002. https://doi.org/10.1103/PhysRevLett.122. 198002

24. Zhu G, Huang Z, Xu Z, Yan LT (2018) Tailoring interfacial nanoparticle organization through entropy. Acc Chem Res 51(4):900909. https://doi.org/10.1021/acs.accounts.8b00001

25. Kirillova A, Marschelke C, Synytska A (2019) Hybrid Janus particles: challenges and opportunities for the design of active functional interfaces and surfaces. ACS Appl Mater Interfaces 11(10): 9643-9671. https://doi.org/10.1021/acsami.8b17709

26. Sheldon RA, Woodley JM (2018) Role of biocatalysis in sustainable chemistry. Chem Rev 118(2):801-838. https://doi.org/10. 1021/acs.chemrev.7b00203

27. Zhao LB, Pan L, Zhang K, Guo SS, Liu W, Wang Y, Chen Y, Zhao XZ, Chan HL (2009) Generation of Janus alginate hydrogel particles with magnetic anisotropy for cell encapsulation. Lab Chip 9(20):2981-2986. https://doi.org/10.1039/b907478c

28. Hu Y, Wang S, Abbaspourrad A, Ardekani AM (2015) Fabrication of shape controllable Janus alginate/pNIPAAm microgels via microfluidics technique and off-chip ionic cross-linking. Langmuir 31(6):1885-1891. https://doi.org/10.1021/la504422j

29. Lee J, Kim J (2012) Multiphasic sensory alginate particle having polydiacetylene liposome for selective and more sensitive multitargeting detection. Chem Mater 24(14):2817-2822. https:// doi.org/10.1021/cm3015012

30. Lan J, Chen J, Li N, Ji X, Yu M, He Z (2016) Microfluidic generation of magnetic-fluorescent Janus microparticles for biomolecular detection. Talanta 151:126-131. https://doi.org/10.1016/j. talanta.2016.01.024

31. Maeda K, Onoe H, Takinoue M, Takeuchi S (2012) Controlled synthesis of 3D multi-compartmental particles with centrifugebased microdroplet formation from a multi-barrelled capillary. Adv Mater 24(10):1340-1346. https://doi.org/10.1002/adma. 201102560

32. Hwang S, Kwak BK, Lee J, Kim DS, Chang ST, Park J, Lee J (2012) Janus hydrogel particles and their aggregation behavior. Macromol Res 20(9):899-901. https://doi.org/10.1007/s13233012-0135-x

33. Wu Q, Yang C, Liu G, Xu W, Zhu Z, Si T, Xu RX (2017) Multiplex coaxial flow focusing for producing multicompartment Janus microcapsules with tunable material compositions and structural characteristics. Lab Chip 17(18):3168-3175. https:// doi.org/10.1039/c7lc00769h

34. Sun X-T, Zhang Y, Zheng D-H, Yue S, Yang C-G, Xu Z-R (2017) Multitarget sensing of glucose and cholesterol based on Janus hydrogel microparticles. Biosens Bioelectron 92:81-86. https:// doi.org/10.1016/j.bios.2017.02.008

35. Jia R, Jiang H, Jin M, Wang X, Huang J (2015) Silver/chitosanbased Janus particles: synthesis, characterization, and assessment of antimicrobial activity in vivo and vitro. Food Res Int 78:433441. https://doi.org/10.1016/j.foodres.2015.08.035

36. Lu AX, Jiang K, DeVoe DL, Raghavan SR (2013) Microfluidic assembly of Janus-like dimer capsules. Langmuir 29(44):13624 13629. https://doi.org/10.1021/la403267j

37. Lu AX, Liu Y, Oh H, Gargava A, Kendall E, Nie Z, DeVoe DL, Raghavan SR (2016) Catalytic propulsion and magnetic steering of soft, patchy microcapsules: ability to pick-up and drop-off microscale cargo. ACS Appl Mater Interfaces 8(24):15676-15683. https://doi.org/10.1021/acsami.6b01245
38. Marquis M, Renard D, Cathala B (2012) Microfluidic generation and selective degradation of biopolymer-based Janus microbeads. Biomacromolecules 13(4):1197-1203. https://doi.org/10.1021/ bm300159u

39. Sheikhi A, van de Ven TGM (2017) Colloidal aspects of Januslike hairy cellulose nanocrystalloids. Curr Opin Colloid Interface Sci 29:21-31. https://doi.org/10.1016/j.cocis.2017.02.001

40. Shao J, Abdelghani M, Shen G, Cao S, Williams DS, van Hest JCM (2018) Erythrocyte membrane modified Janus polymeric motors for thrombus therapy. ACS Nano 12(5):4877-4885. https://doi.org/10.1021/acsnano.8b01772

41. Schattling PS, Ramos-Docampo MA, Salgueiriño V, Städler B (2017) Double-fueled Janus swimmers with magnetotactic behavior. ACS Nano 11(4):3973-3983. https://doi.org/10.1021/ acsnano.7b00441

42. Gao W, Feng X, Pei A, Gu Y, Li J, Wang J (2013) Seawater-driven magnesium based Janus micromotors for environmental remediation. Nanoscale 5(11):4696-4700. https://doi.org/10.1039/ c3nr01458d

43. Lim YGJ, Poh KCW, Loo SCJ (2019) Hybrid Janus microparticles achieving selective encapsulation for theranostic applications via a facile solvent emulsion method. Macromol Rapid Commun 40(7):e1800801. https://doi.org/10.1002/marc.201800801

44. Ekanem EE, Nabavi SA, Vladisavljevic GT, Gu S (2015) Structured biodegradable polymeric microparticles for drug delivery produced using flow focusing glass microfluidic devices. ACS Appl Mater Interfaces 7(41):23132-23143. https://doi.org/10. 1021/acsami.5b06943

45. Bhaskar S, Gibson CT, Yoshida M, Nandivada H, Deng X, Voelcker NH, Lahann J (2011) Engineering, characterization and directional self-assembly of anisotropically modified nanocolloids. Small 7(6):812-819. https://doi.org/10.1002/smll. 201001695

46. Garbuzenko OB, Winkler J, Tomassone MS, Minko T (2014) Biodegradable Janus nanoparticles for local pulmonary delivery of hydrophilic and hydrophobic molecules to the lungs. Langmuir 30(43):12941-12949. https://doi.org/10.1021/la502144z

47. Matsumoto A, Murao S, Matsumoto M, Watanabe C, Murakami M (2016) Fabrication of Janus particles composed of poly (lacticco-glycolic) acid and hard fat using a solvent evaporation method. Drug Discoveries \& Therapeutics 10(6):307-313. https://doi.org/ $10.5582 /$ ddt.2016.01230

48. Xie H, She Z-G, Wang S, Sharma G, Smith JW (2012) One-step fabrication of polymeric Janus nanoparticles for drug delivery. Langmuir 28(9):4459-4463. https://doi.org/10.1021/la2042185

49. Li P, Li K, Niu X, Fan Y (2016) Electrospraying magneticfluorescent bifunctional Janus PLGA microspheres with dual rare earth ions fluorescent-labeling drugs. RSC Adv 6(101):99034 99043. https://doi.org/10.1039/C6RA15401H

50. Ekanem EE, Zhang Z, Vladisavljevic GT (2017) Facile production of biodegradable bipolymer patchy and patchy Janus particles with controlled morphology by microfluidic routes. Langmuir 33(34):8476-8482. https://doi.org/10.1021/acs.langmuir.7b02506

51. Kim SW, Hwangbo K-H, Lee JH, Cho KY (2014) Microfluidic fabrication of microparticles with multiple structures from a biodegradable polymer blend. RSC Adv 4(87):46536-46540. https:// doi.org/10.1039/c4ra05864j

52. Zhao Z, Zhu F, Qu X, Wu Q, Wang Q, Zhang G, Liang F (2015) pH-responsive polymeric Janus containers for controlled drug delivery. Polym Chem 6(22):4144-4153. https://doi.org/10.1039/ c5py00267b

53. Li W, Dong H, Tang G, Ma T, Cao X (2015) Controllable microfluidic fabrication of Janus and microcapsule particles for drug delivery applications. RSC Adv 5(30):23181-23188. https:// doi.org/10.1039/C4RA17153E 
54. Han D, Xiao P, Gu J, Chen J, Cai Z, Zhang J, Wang W, Chen T (2014) Polymer brush functionalized Janus graphene oxide/ chitosan hybrid membranes. RSC Adv 4(43):22759. https://doi. org/10.1039/c4ra02826k

55. Kovach I, Won J, Friberg SE, Koetz J (2016) Completely engulfed olive/silicone oil Janus emulsions with gelatin and chitosan. Colloid Polym Sci 294(4):705-713. https://doi.org/10.1007/ s00396-016-3828-4

56. Raju RR, Kosmella S, Friberg SE, Koetz J (2017) Pickering Janus emulsions and polyelectrolyte complex-stabilized Janus gels. Colloids Surf A Physicochem Eng Asp 533:241-248. https://doi. org/10.1016/j.colsurfa.2017.08.022

57. Khoee S, Mansouri Bakvand P (2019) Synthesis of dualresponsive Janus nanovehicle via PNIPAm modified SPIONs deposition on crosslinked chitosan microparticles and decrosslinking process in the core. Eur Polym J 114:411-425. https://doi.org/10.1016/j.eurpolymj.2019.03.007

58. Seiffert S, Romanowsky MB, Weitz DA (2010) Janus microgels produced from functional precursor polymers. Langmuir 26(18): 14842-14847. https://doi.org/10.1021/la101868w

59. Hwang DK, Oakey J, Toner M, Arthur JA, Anseth KS, Lee S, Zeiger A, Van Vliet KJ, Doyle PS (2009) Stop-flow lithography for the production of shape-evolving degradable microgel particles. J Am Chem Soc 131(12):4499-4504. https://doi.org/10. 1021/ja809256d

60. Suh SK, Yuet K, Hwang DK, Bong KW, Doyle PS, Hatton TA (2012) Synthesis of nonspherical superparamagnetic particles: in situ coprecipitation of magnetic nanoparticles in microgels prepared by stop-flow lithography. J Am Chem Soc 134(17):73377343. https://doi.org/10.1021/ja209245v

61. Seo KD, Doh J, Kim DS (2013) One-step microfluidic synthesis of Janus microhydrogels with anisotropic thermo-responsive behavior and organophilic/hydrophilic loading capability. Langmuir 29(49):15137-15141. https://doi.org/10.1021/la403015y

62. Ma S, Thiele J, Liu X, Bai Y, Abell C, Huck WTS (2012) Fabrication of microgel particles with complex shape via selective polymerization of aqueous two-phase systems. Small 8(15):23562360. https://doi.org/10.1002/smll.201102715

63. Chen Q, Zheng L, Chen B, He J, Huang H, Lin J (2014) Highly efficient phase transfer catalyst supported on Janus composite particles: synthesis, characterization, and applications. J Mater Res 29(11):1231-1236. https://doi.org/10.1557/jmr.2014.117

64. Kirillova A, Schliebe C, Stoychev G, Jakob A, Lang H, Synytska A (2015) Hybrid hairy Janus particles decorated with metallic nanoparticles for catalytic applications. ACS Appl Mater Interfaces 7(38):21218-21225. https://doi.org/10.1021/acsami. 5 b05224

65. Wang J, Huang R, Qi W, Su R, He Z (2017) Oriented enzyme immobilization at the oil/water interface enhances catalytic activity and recyclability in a Pickering emulsion. Langmuir 33(43): 12317-12325. https://doi.org/10.1021/acs.langmuir.7b02862

66. Xu X, Liu Y, Gao Y, Li H (2017) Preparation of Au@ silica Janus nanosheets and their catalytic application. Colloids Surf A Physicochem Eng Asp 529:613-620. https://doi.org/10.1016/j. colsurfa.2017.06.048

67. Shinde P, Gupta SS, Singh B, Polshettiwar V, Prasad Bhagavatula LV (2017) Amphi-functional mesoporous silica nanoparticles for dye separation. J Mater Chem A 5(28):14914-14921. https://doi. org/10.1039/c7ta03904b

68. Kong X, Wu C, Feng L, Qu J, Liu P, Wang X, Zhang X (2017) Silica-based hierarchical porous Janus microcapsules: construction and support of Au nano-particle catalyst inside. Chem Commun (Camb) 53(57):8054-8057. https://doi.org/10.1039/ c7cc03077k

69. Liu J, Wang P, Zhou M, Ma Y, Niu X, Pan G, Pan J (2019) Tailored Janus silica nanosheets integrating bispecific artificial receptors for simultaneous adsorption of 2,6-dichlorophenol and $\mathrm{Pb}$ (ii). J Mater Chem A 7(27):16161-16175. https://doi.org/10. 1039/c9ta01116a

70. Tang X, Hou Y, Meng QB, Zhang G, Liang F, Song X-M (2019) Heteropoly acids-functionalized Janus particles as catalytic emulsifier for heterogeneous acylation in flow ionic liquid-in-oil Pickering emulsion. Colloids Surf A Physicochem Eng Asp 570: 191-198. https://doi.org/10.1016/j.colsurfa.2019.02.066

71. Dou S-Y, Wang R (2019) The C-Si Janus nanoparticles with supported phosphotungstic active component for Pickering emulsion desulfurization of fuel oil without stirring. Chem Eng J 369:64 76. https://doi.org/10.1016/j.cej.2019.03.050

72. Zhang M, Tang Z, Fu W, Wang W, Tan R, Yin D (2019) An ionic liquid-functionalized amphiphilic Janus material as a Pickering interfacial catalyst for asymmetric sulfoxidation in water. Chem Commun (Camb) 55(5):592-595. https://doi.org/10.1039/ $\mathrm{c} 8 \mathrm{cc} 08292 \mathrm{~h}$

73. Yi F, Xu F, Gao Y, Li H, Chen D (2015) Macrocellular polymer foams from water in oil high internal phase emulsion stabilized solely by polymer Janus nanoparticles: preparation and their application as support for Pd catalyst. RSC Adv 5(50):4022740235. https://doi.org/10.1039/c5ra01859e

74. Ali N, Zhang B, Zhang H, Li W, Zaman W, Tian L, Zhang Q (2015) Novel Janus magnetic micro particle synthesis and its applications as a demulsifier for breaking heavy crude oil and water emulsion. Fuel 141:258-267. https://doi.org/10.1016/j.fuel.2014. 10.026

75. Cho J, Cho J, Kim H, Lim M, Jo H, Kim H, Min S-J, Rhee H, Kim JW (2018) Janus colloid surfactant catalysts for in situ organic reactions in Pickering emulsion microreactors. Green Chem 20(12):2840-2844. https://doi.org/10.1039/c8gc00282g

76. Jia L, Zhou T, Xu J, Li X, Dong K, Huang J, Xu Z (2016) The enhanced catalytic activities of asymmetric Au-Ni nanoparticle decorated halloysite-based nanocomposite for the degradation of organic dyes. Nanoscale Res Lett 11(1):72. https://doi.org/10. 1186/s11671-016-1252-9

77. Ku KH, Lee YJ, Yi G-R, Jang SG, Schmidt BVKJ, Liao K, Klinger D, Hawker CJ, Kim BJ (2017) Shape-tunable biphasic Janus particles as $\mathrm{pH}$-responsive switchable surfactants. Macromolecules 50(23):9276-9285. https://doi.org/10.1021/acs. macromol.7b02365

78. Jia X, Ma Y, Liu Y, Zhang B, Zhang H, Zhang Q (2018) Quaternary ammonium functionalized Fe3O4 \& P(GMA-AADVB) magnetic Janus particles as highly efficient catalysts for phase transfer reactions. Dalton Trans 47(37):12893-12900. https://doi.org/10.1039/c8dt02433b

79. Zhao T, Zhu X, Hung CT, Wang P, Elzatahry A, Al-Khalaf AA, Hozzein WN, Zhang F, Li X, Zhao D (2018) Spatial isolation of carbon and silica in a single Janus mesoporous nanoparticle with tunable amphiphilicity. J Am Chem Soc 140(31):10009-10015. https://doi.org/10.1021/jacs.8b06127

80. Yao J, Ma Y, Liu J, Liu S, Pan J (2019) Janus-like boronate affinity magnetic molecularly imprinted nanobottles for specific adsorption and fast separation of luteolin. Chem Eng J 356:436-444. https://doi.org/10.1016/j.cej.2018.09.003

81. Xue D, Meng QB, Song XM (2019) Magnetic-responsive Janus nanosheets with catalytic properties. ACS Appl Mater Interfaces 11(11):10967-10974. https://doi.org/10.1021/acsami.8b21012

82. Pan D, Mou F, Li X, Deng Z, Sun J, Xu L, Guan J (2016) Multifunctional magnetic oleic acid-coated MnFe2O4/ polystyrene Janus particles for water treatment. J Mater Chem A 4(30):11768-11774. https://doi.org/10.1039/c6ta04010a

83. Ren M, Guo W, Guo H, Ren X (2019) Microfluidic fabrication of bubble-propelled micromotors for wastewater treatment. ACS Appl Mater Interfaces 11(25):22761-22767. https://doi.org/10. 1021/acsami.9b05925 
84. Zhao R, Han T, Sun D, Huang L, Liang F, Liu Z (2019) Poly(ionic liquid)-modified magnetic Janus particles for dye degradation. Langmuir 35(35):11435-11442. https://doi.org/10.1021/acs. langmuir.9b01400

85. Matura A, Köpke D, Marschelke C, Kramer J, Synytska A, Sallat $\mathrm{M}$ (submitted) Functional core-shell particles for enzyme immobilization and their application

86. Gao W, Wang J (2014) The environmental impact of micro/ nanomachines: a review. ACS Nano 8(4):3170-3180. https://doi. org/10.1021/nn500077a

87. Qu X, Brame J, Li Q, Alvarez PJJ (2013) Nanotechnology for a safe and sustainable water supply: enabling integrated water treatment and reuse. Acc Chem Res 46(3):834-843. https://doi.org/10. $1021 / \operatorname{ar} 300029 \mathrm{v}$

88. Kang DH, Jung HS, Ahn N, Yang SM, Seo S, Suh KY, Chang PS, Jeon NL, Kim J, Kim K (2014) Janus-compartmental alginate microbeads having polydiacetylene liposomes and magnetic nanoparticles for visual lead(II) detection. ACS Appl Mater Interfaces 6(13):10631-10637. https://doi.org/10.1021/ am502319m

89. Chun HJ, Kim S, Han YD, Kim DW, Kim KR, Kim H-S, Kim J$\mathrm{H}$, Yoon HC (2018) Water-soluble mercury ion sensing based on the thymine-Hg2+-thymine base pair using retroreflective Janus particle as an optical signaling probe. Biosens Bioelectron 104: 138-144. https://doi.org/10.1016/j.bios.2018.01.008

90. Wang Y, Shang M, Wang Y, Xu Z (2019) Droplet-based microfluidic synthesis of (Au nanorod@Ag)-polyaniline Janus nanoparticles and their application as a surface-enhanced Raman scattering nanosensor for mercury detection. Anal Methods 11(31):3966-3973. https://doi.org/10.1039/C9AY01213C

91. Wu J, Balasubramanian S, Kagan D, Manesh KM, Campuzano S, Wang J (2010) Motion-based DNA detection using catalytic nanomotors. Nat Commun 1:36. https://doi.org/10.1038/ ncomms 1035

92. Simmchen J, Baeza A, Ruiz D, Esplandiu MJ, Vallet-Regi M (2012) Asymmetric hybrid silica nanomotors for capture and cargo transport: towards a novel motion-based DNA sensor. Small 8(13):2053-2059. https://doi.org/10.1002/smll.201101593

93. Campuzano S, Orozco J, Kagan D, Guix M, Gao W, Sattayasamitsathit S, Claussen JC, Merkoci A, Wang J (2012) Bacterial isolation by lectin-modified microengines. Nano Lett 12(1):396-401. https://doi.org/10.1021/nl203717q

94. Pacheco M, Jurado-Sanchez B, Escarpa A (2018) Sensitive monitoring of Enterobacterial contamination of food using selfpropelled Janus microsensors. Anal Chem 90(4):2912-2917. https://doi.org/10.1021/acs.analchem.7b05209

95. Zhao L, Xie S, Liu Y, Liu Q, Song X, Li X (2019) Janus micromotors for motion-capture-lighting of bacteria. Nanoscale. https://doi.org/10.1039/c9nr05503g

96. Molinero-Fernandez A, Moreno-Guzman M, Lopez MA, Escarpa A (2017) Biosensing strategy for simultaneous and accurate quantitative analysis of mycotoxins in food samples using unmodified graphene micromotors. Anal Chem 89(20):10850-10857. https:// doi.org/10.1021/acs.analchem.7b02440

97. Zheng F, Ke W, Shi L, Liu H, Zhao Y (2019) Plasmonic Au-Ag Janus nanoparticle engineered ratiometric surface-enhanced Raman scattering aptasensor for ochratoxin A detection. Anal Chem. https://doi.org/10.1021/acs.analchem.9b02469

98. Yang Y-J, Zhou Y, Xing Y, Zhang G-M, Zhang Y, Zhang C-H, Lei P, Dong C, Deng X, He Y, S-m S (2019) A label-free aptasensor based on Aptamer/NH2 Janus particles for ultrasensitive electrochemical detection of ochratoxin A. Talanta 199:310-316. https:// doi.org/10.1016/j.talanta.2019.02.015

99. Fujishima A, Rao TN, Tryk DA (2000) Titanium dioxide photocatalysis. J Photochem Photobiol C: Photochem Rev 1(1): 1-21. https://doi.org/10.1016/S1389-5567(00)00002-2
100. Huang H, Chen Q, He J, Zheng L, Chen B, Lin J (2014) Synthesis and characterization of $\mathrm{TiO} 2 / \mathrm{C}$ Janus composite particles and its photocatalytic activity for the degradation of rhodamine B. Colloid Polym Sci 292(12):3085-3093. https://doi.org/10.1007/ s00396-014-3362-1

101. Panwar K, Jassal M, Agrawal AK (2016) TiO2-SiO2 Janus particles with highly enhanced photocatalytic activity. RSC Adv 6(95):92754-92764. https://doi.org/10.1039/c6ra12378c

102. Jurado-Sanchez B, Sattayasamitsathit S, Gao W, Santos L, Fedorak Y, Singh VV, Orozco J, Galarnyk M, Wang J (2015) Self-propelled activated carbon Janus micromotors for efficient water purification. Small 11(4):499-506. https://doi.org/10.1002/ smll.201402215

103. Mou F, Kong L, Chen C, Chen Z, Xu L, Guan J (2016) Lightcontrolled propulsion, aggregation and separation of water-fuelled $\mathrm{TiO} 2 / \mathrm{Pt}$ Janus submicromotors and their "on-the-fly" photocatalytic activities. Nanoscale 8(9):4976-4983. https://doi.org/10. $1039 / \mathrm{c} 5 \mathrm{nr} 06774 \mathrm{j}$

104. Orozco J, Mercante LA, Pol R, Merkoçi A (2016) Graphene-based Janus micromotors for the dynamic removal of pollutants. J Mater Chem A 4(9):3371-3378. https://doi.org/10.1039/c5ta09850e

105. Lee C-S, Gong J, Oh D-S, Jeon J-R, Chang Y-S (2018) Zerovalent-iron/platinum Janus micromotors with spatially separated functionalities for efficient water decontamination. ACS Appl Nano Mater 1(2):768-776. https://doi.org/10.1021/ acsanm.7b00223

106. Wang J, Dong R, Yang Q, Wu H, Bi Z, Liang Q, Wang Q, Wang C, Mei Y, Cai Y (2019) One body, two hands: photocatalytic function- and Fenton effect-integrated light-driven micromotors for pollutant degradation. Nanoscale. https://doi.org/10.1039/ c9nr04295d

107. Pourrahimi AM, Villa K, Ying Y, Sofer Z, Pumera M (2018) ZnO/ $\mathrm{ZnO} 2 / \mathrm{Pt}$ Janus micromotors propulsion mode changes with size and Interface structure: enhanced nitroaromatic explosives degradation under visible light. ACS Appl Mater Interfaces 10(49): 42688-42697. https://doi.org/10.1021/acsami.8b16217

108. Kong L, Ambrosi A, Nasir MZM, Guan J, Pumera M (2019) Selfpropelled 3D-printed "aircraft carrier" of light-powered smart micromachines for large-volume nitroaromatic explosives removal. Advanced Functional Materials:1903872. doi:https://doi.org/ 10.1002/adfm.201903872

109. Luo D, Wang F, Zhu J, Cao F, Liu Y, Li X, Willson RC, Yang Z, Chu CW, Ren Z (2016) Nanofluid of graphene-based amphiphilic Janus nanosheets for tertiary or enhanced oil recovery: high performance at low concentration. Proc Natl Acad Sci U S A 113(28): 7711-7716. https://doi.org/10.1073/pnas.1608135113

110. Luo D, Wang F, Zhu J, Tang L, Zhu Z, Bao J, Willson RC, Yang Z, Ren Z (2017) Secondary oil recovery using graphene-based amphiphilic Janus nanosheet fluid at an ultralow concentration. Ind Eng Chem Res 56(39):11125-11132. https://doi.org/10.1021/acs. iecr.7b02384

111. Yin T, Yang Z, Dong Z, Lin M, Zhang J (2019) Physicochemical properties and potential applications of silica-based amphiphilic Janus nanosheets for enhanced oil recovery. Fuel 237:344-351. https://doi.org/10.1016/j.fuel.2018.10.028

112. Liang F, Shen K, Qu X, Zhang C, Wang Q, Li J, Liu J, Yang Z (2011) Inorganic Janus nanosheets. Angew Chem Int Ed Eng 50(10):2379-2382. https://doi.org/10.1002/anie.201007519

113. Song Y, Zhou J, Fan J-B, Zhai W, Meng J, Wang S (2018) Hydrophilic/oleophilic magnetic Janus particles for the rapid and efficient oil-water separation. Adv Funct Mater 28(32):1802493. https://doi.org/10.1002/adfm.201802493

114. Soto F, Lopez-Ramirez MA, Jeerapan I, Esteban-Fernandez de Avila B, Mishra RK, Lu X, Chai I, Chen C, Kupor D, Nourhani A, Wang J (2019) Rotibot: use of rotifers as self-propelling 
biohybrid microcleaners. Adv Funct Mater 29(22):1900658. https://doi.org/10.1002/adfm.201900658

115. Hwang J, Yang H-M, Lee K-W, Jung Y-I, Lee KJ, Park CW (2019) A remotely steerable Janus micromotor adsorbent for the active remediation of Cs-contaminated water. J Hazard Mater 369:416422. https://doi.org/10.1016/j.jhazmat.2019.02.054

116. Kadam R, Maas M, Rezwan K (2019) Selective, agglomerate-free separation of bacteria using biofunctionalized, magnetic Janus nanoparticles. ACS Appl Bio Mater 2(8):3520-3531. https://doi. org/10.1021/acsabm.9b00415

117. Wang L, Kaeppler A, Fischer D, Simmchen J (2019) Photocatalytic $\mathrm{TiO} 2$ micromotors for removal of microplastics and suspended matter. ACS Appl Mater Interfaces 11(36): 32937-32944. https://doi.org/10.1021/acsami.9b06128

118. Yuan Q, Liu D, Zhang N, Ye W, Ju H, Shi L, Long R, Zhu J, Xiong Y (2017) Noble-metal-free Janus-like structures by cation exchange for Z-scheme photocatalytic water splitting under broadband light irradiation. Angew Chem Int Ed Eng 56(15):42064210. https://doi.org/10.1002/anie.201700150

119. Chauhan A, Rastogi M, Scheier P, Bowen C, Kumar RV, Vaish R (2018) Janus nanostructures for heterogeneous photocatalysis. Appl Phys Rev 5(4):041111. https://doi.org/10.1063/1.5039926

120. Zhang J, Jin X, Morales-Guzman PI, Yu X, Liu H, Zhang H, Razzari L, Claverie JP (2016) Engineering the absorption and field enhancement properties of $\mathrm{Au}-\mathrm{TiO} 2$ nanohybrids via whispering gallery mode resonances for photocatalytic water splitting. ACS Nano 10(4):4496-4503. https://doi.org/10.1021/acsnano.6b00263

121. Liu X, Xuan Y (2017) Full-spectrum volumetric solar thermal conversion via photonic nanofluids. Nanoscale 9(39):14854 14860. https://doi.org/10.1039/c7nr03912c

122. Si Y, Cao S, Wu Z, Ji Y, Mi Y, Wu X, Liu X, Piao L (2017) The effect of directed photogenerated carrier separation on photocatalytic hydrogen production. Nano Energy 41:488-493. https://doi. org/10.1016/j.nanoen.2017.10.008

123. Wang C, Bu E, Chen Y, Cheng Z, Zhang J, Shu R, Song Q (2019) Enhanced photoreforming hydrogen production: Pickering interfacial catalysis from a bio-derived biphasic system. Renew Energy 134:113-124. https://doi.org/10.1016/j.renene.2018.09.001

124. Bu E, Chen Y, Wang C, Cheng Z, Luo X, Shu R, Zhang J, Liao M, Jiang Z, Song Q (2019) Hydrogen production from bio-derived biphasic photoreforming over a raspberry-like amphiphilic $\mathrm{Ag} 2 \mathrm{O}$ TiO2/SiO2 catalyst. Chem Eng J 370:646-657. https://doi.org/10. 1016/j.cej.2019.03.259

125. Sakata Y, Hirata Y, Miyahara K, Imamura H, Tsuchiya S (1993) Photocatalytic activity of titanium dioxide loaded with cerium oxide. Chem Lett 22(3):391-394. https://doi.org/10.1246/cl. 1993.391

126. Seh ZW, Liu S, Low M, Zhang SY, Liu Z, Mlayah A, Han MY (2012) Janus Au-TiO2 photocatalysts with strong localization of plasmonic near-fields for efficient visible-light hydrogen generation. Adv Mater 24(17):2310-2314. https://doi.org/10.1002/adma. 201104241

Publisher's note Springer Nature remains neutral with regard to jurisdictional claims in published maps and institutional affiliations.

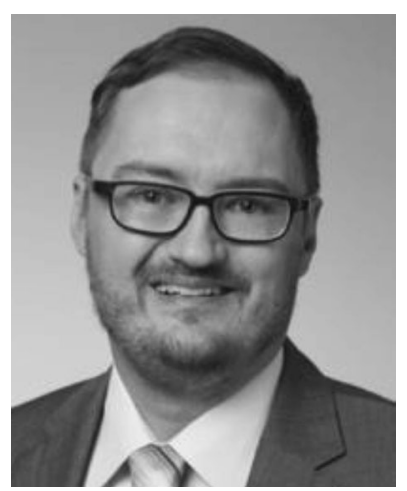

Andreas Fery I studied Physics at Konstanz University and received my $\mathrm{PhD}$ at Potsdam University in 2000. After a post-doc at Institute Curie Paris, I became group leader at Max-Planck Institute for colloids and interfaces. In 2007 I joined Bayreuth University as professor for Physical Chemistry, where Matthias Ballauff established the CRC 840 "From particulate systems to mesotechnology", my scientific home for the following 8 years. Since 2015 I joined the Leibniz Institut für Polymerforschung Dresden, where Matthias serves as member of the scientific advisory board. I got to know Matthias as a very supportive colleague, who is always eager to help scientists develop their potential and an inspirational leader of our research field with whom I share the passion for colloid and interface science. I highly appreciate his sense of humor and rhetoric gift that allows him to get to the point even like few others can. All the very best for your future.

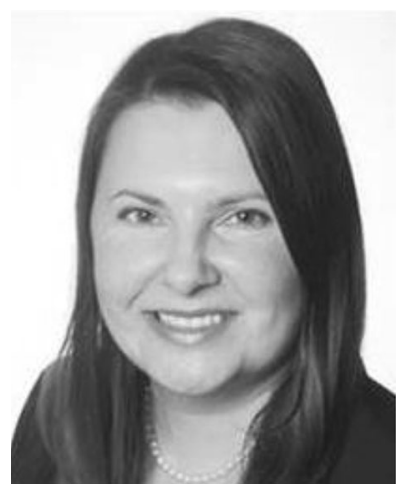

Alla Synytska I studied Chemistry at the Ivan Franko National University of Lviv in Ukraine and received my $\mathrm{PhD}$ from the Technical University of Dresden in 2005. After a postdoctoral training at the University of Modena and at the ESPCI Paris Tech, I became an independent research group leader (Functional Particles and Interfaces) at the Leibniz Institute of Polymer Research Dresden (IPF) in 2012. I received my habilitation degree from the Technical University of Dresden in 2018. I currently lead the Department of Polymer Interfaces at the IPF and teach at the Technical University of Dresden as a Privatdozent. Professor Matthias Ballauff was my mentor during my habilitation within the Leibniz-Mentoring Program. I am incredibly thankful to Matthias for his extensive mentoring, reflection about my research and teaching as well as sharing his personal experience and competences with me. I owe my deepest gratitude to you, Matthias, for your guidance, endless support, inspiration and trust in me and my research on Janus colloids and interfaces. I wish you all the best and much success for your future. 